\title{
MAXIMAL ABELIAN SUBALGEBRAS OF VON NEUMANN ALGEBRAS AND REPRESENTATIONS OF EQUIVALENCE RELATIONS \\ BY
}

\author{
COLIN E. SUTHERLAND
}

\begin{abstract}
We associate to each pair $(\mathfrak{R}, \mathbb{Q}$ ), (with $\mathfrak{T}$ a von Neumann algebra, and $Q$ a maximal abelian subalgebra) a representation $\alpha$ of the Takesaki equivalence relation $\Re(\Re, \mathbb{Q})$ of $(\Re, Q)$ as automorphisms of a $I_{\infty}$ factor. Conversely each such representation $\alpha$ of $\Re$ on $(X, \mu)$ as automorphisms of $\mathfrak{B}(\mathcal{K})$ determines a von Neumann algebra-abelian subalgebra pair $S^{\prime}(\Re, \alpha)=(\mathscr{R}, \mathscr{B})$ where $\mathscr{R}$ is the commutant of the algebra of "self-intertwiners" for $\alpha$ and $\mathscr{B}=L^{\infty}(X, \mu) \otimes 1$ on $L^{2}(X, \mu) \otimes \mathcal{H}$. The main concern is the assignments $(\mathfrak{R}, \mathbb{Q}) \rightarrow \mathscr{T}(\mathscr{T}, \mathbb{Q})=$ $(\Re(\Re, Q), \alpha)$ and $(\Re, \alpha) \rightarrow S^{\prime}(\Re, \alpha)$, and in particular, the extent to which they are inverse to each other-this occurs if $R$ is countable nonsingular and $\alpha$ is (conjugation by) a projective square-integrable representation (cf. [8]), or if $\mathbb{Q}$ is a Cartan subalgebra (cf. [5]), among other cases. A partial dictionary between the representations $(R, \alpha)$ and pairs $(\Re, \mathscr{Q})$ is given - thus if $R$ is countable nonsingular and $\alpha$ is what we term replete, $S^{\prime}(\mathscr{R}, \alpha)$ is injective whenever $R$ is amenable, and a complete Galois theory generalizing that for crossed products by discrete groups is available. We also show how to construct various pathological examples such as a singular maximal abelian subalgebra $Q \subseteq \mathfrak{K}$ for which the Takesaki equivalence relation $\mathscr{R}(\mathfrak{R}, \mathcal{Q})$ is nontrivial.
\end{abstract}

Introduction. This paper introduces and studies a special kind of maximal abelian subalgebra for von Neumann algebras. These subalgebras, which we term complete, are proper generalizations of regular abelian subalgebras (i.e. those whose normalizers in the ambient von Neumann algebra generate the ambient algebra), and yet are, in many respects, as well behaved and determining as regular abelian subalgebras are.

Throughout, a fundamental role is played by the notion of a (measurable) representation $\alpha$ of an equivalence relation $\mathcal{R}$ on a measure space $(\Gamma, \mu)$ as automorphisms of the type $\mathrm{I}_{\infty}$ factor $\mathfrak{B}\left(\mathcal{H}_{0}\right)$. Such a representation is a Borel map $\alpha:\left(\gamma, \gamma^{\prime}\right) \in \mathscr{R} \rightarrow \alpha\left(\gamma, \gamma^{\prime}\right) \in \operatorname{Aut}\left(\mathfrak{B}\left(\mathcal{H}_{0}\right)\right)$ with $\alpha\left(\gamma, \gamma^{\prime}\right) \alpha\left(\gamma^{\prime}, \gamma^{\prime \prime}\right)=\alpha\left(\gamma, \gamma^{\prime \prime}\right)$ for $\left(\gamma, \gamma^{\prime \prime}\right),\left(\gamma^{\prime}, \gamma^{\prime \prime}\right) \in \mathcal{R}$. Each representation $(\mathcal{R}, \alpha)$ determines a von Neumann algebra-abelian subalgebra pair $(\mathfrak{N}, \mathcal{Q})=S^{\prime}(\Re, \alpha)$, where $\Re$ is the commutant of the "algebra of sections" of $\alpha$, and $Q$ is isomorphic with $L^{\infty}(\Gamma, \mu)$ (both acting on $\left.L^{2}(\Gamma, \mu) \otimes \mathcal{H}_{0}\right)$. Conversely, to each von Neumann algebra-maximal abelian subalgebra pair $(\mathfrak{R}, \mathbb{Q})$, Takesaki has associated an equivalence relation $\Re(\Re, \mathbb{Q})$; we

Received by the editors August 20, 1982.

1980 Mathematics Subject Classification. Primary 46L10, 20 L99.

Key words and phrases. Maximal abelian subalgebras, Cartan subalgebra, groupoid cohomology, transverse measure. 


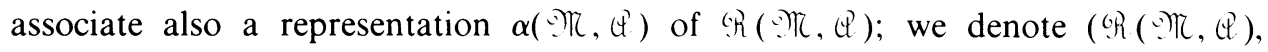
$\alpha(\mathscr{N}, \mathbb{Q}))$ by $\widetilde{\mathcal{T}}(\mathfrak{O}, \mathbb{Q})$.

The complete abelian subalgebras are those pairs ( $), Q$ ) for which $S^{\prime}(\bar{T}(\mathcal{O}, \mathbb{Q})$ ) $=(\mathcal{O}, \mathbb{Q})$; the paper then is concerned with the interplay between complete pairs $(\mathcal{O}, Q)$ and representations $\widetilde{T}(\mathcal{N}, \mathcal{Q}) . \S 1$ is preliminary, while $\S 2$ investigates elementary properties of the assignment $(\Re, \alpha) \rightarrow S^{\prime}(\Re, \alpha)$. In $\S 3$ we introduce the Takesaki relation alluded to above, and also the notion of completeness for pairs $(\mathcal{O}, \mathbb{Q})$, and, dually, for pairs $(\Re, \alpha)$. Every pair $(\mathcal{M}, \mathbb{Q})$ or $(\Re, \alpha)$ is shown to have a canonical completion. We also give necessary and sufficient conditions for conjugacy of complete maximal abelian subalgebras, and an example of a pair $(\mathcal{O}, Q$ ) where $Q$ is singular maximal abelian, but for which $\Re(\mathcal{R}, Q$ ) is not trivial, thus answering a question raised by Takesaki.

In $\$ 4$ we establish a partial dictionary between pairs $(\mathcal{M}, \mathscr{Q})$ and $(\mathscr{R}, \alpha)$; thus $Q \subseteq \subseteq$ is regular if and only if $(\Re, Q)=S^{\prime}(\Re, \alpha)$ for some representation of some equivalence relation $\Omega$ with countable equivalence classes and which is nonsingular in a suitable sense. We characterize those $(\mathfrak{N}, \mathbb{Q}$ ) with $\Re$ type $I$ or a factor in terms of $\mathscr{R}(\mathcal{M}, Q)$, and show that amenability $\mathscr{R}$ in the sense of Zimmer implies injectivity of the algebras $S(\Re, \alpha)^{\prime}$. In $\S 5$ certain concrete representations $\tau^{\omega}$ of $R$ are given; these are analogues of the left regular projective representations of groups, and they are used to give necessary and sufficient conditions for $\mathcal{Q} \subseteq$ Y to be a Cartan subalgebra in the terminology of Feldman and Moore. Specifically, $\mathbb{Q} \subseteq \mathcal{N}$ is Cartan if and only if it is complete and $\alpha(\mathscr{N}, \mathbb{Q})$ is equivalent in a suitable sense to $\tau^{\omega}$ for an appropriate $\omega \in H^{2}(\Re, T)$. Finally, $\S 6$ gives a Galois correspondence

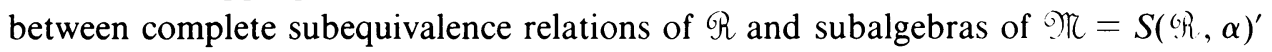
which are intermediate between $\mathcal{Q}$ and $\mathcal{M}$ and which are complete (over $\mathcal{Q}$ ).

This work clearly has much in common with other recent papers; in particular, the notion of "algebra of sections" generalizes the more familiar crossed products (see $[13,15])$ as well as the idea of extensions of [13]; this notion was suggested by Mackey in [10] and used in a context similar to ours in [8]. This paper originated in an attempt to understand better regular subalgebras, and clearly owes much to the work of Feldman and Moore in $[4,5]$. Also, to some extent, our results complement recent work of Connes on noncommutative integration - see Springer Lecture Notes, vol. 725-especially in $\$ 4$ where we consider "countable nonsingular" equivalence relations $(\mathcal{R}, \Gamma, \mu)$. Even so, for our purpose, it is vital to consider measures $\mu$ which are not nonsingular, and hence not of the form $\Lambda_{\nu}$ for any transverse measure $\Lambda$ and transverse function $\nu$ on $\Re$.

\section{Representations of equivalence relations.}

1. Preliminaries. Let $\Gamma$ be a standard Borel space; we shall be considering analytic equivalence relations on $\Gamma$, i.e. equivalence relations $\Re \subseteq \Gamma \times \Gamma$ which are analytic as subsets of $\Gamma \times \Gamma$, together with Borel measures $\mu$ on $\Gamma$. We refer to $(\mathcal{R}, \Gamma, \mu)$ as an analytic equivalence relation on $(\Gamma, \mu)$. For $\gamma \in \Gamma$, set $R(\gamma)=\left\{\gamma^{\prime}:\left(\gamma, \gamma^{\prime}\right) \in \Re\right\}$, and for $E \subseteq \Gamma$, set $\Re(E)=\bigcup\{\Re(\gamma): \gamma \in E\}$, the saturation of $E$. Since $\Re$ is analytic, $R(E)$ is analytic whenever $E$ is (see [11] for undefined terminology). 
For $E \subseteq \Gamma$, set $\left.\bullet R\right|_{E}=\left(R \cap(E \times E)\right.$; $\left.R\right|_{E}$ is the reduction of $h$ by $E$; if $E$ is conull (i.e. $\mu(\Gamma-E)=0),\left.\stackrel{\leftrightarrow}{R}\right|_{E}$ is an inessential reduction (abbreviated i.r.) of $(h$.

Definition 1.1. Analytic equivalence relations $(\Re, \Gamma, \mu)$ and $(\Omega, \Omega, \nu)$ are isomorphic if there are inessential reductions $\left.h\right|_{E},\left.s\right|_{F}$ and a Borel bijection $\Phi: E \rightarrow F$ with $\nu \cdot \Phi$ equivalent to $\mu$ and $\left.\left(\gamma, \gamma^{\prime}\right) \in \Re\right|_{E}$ if and only if $\left.\left(\Phi(\gamma), \Phi\left(\gamma^{\prime}\right)\right) \in S\right|_{F}$.

The group of all automorphisms of $($ ? $R, \Gamma, \mu)$ is denoted $\operatorname{Aut}(\Re, \mu)$; isomorphisms are to be identified if they agree on some i.r. Those automorphisms $\Phi$ of

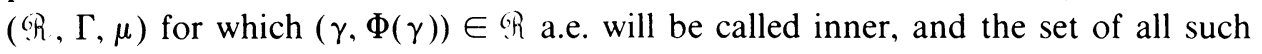
denoted Int $(\Re, \mu)$.

Throughout, $\mathfrak{K}_{0}$ denotes a fixed, separable, infinite dimensional Hilbert space, $\mathfrak{B}\left(\mathcal{H}_{0}\right)$ the bounded operators on $\mathfrak{H}_{0}$, and $\operatorname{Aut}\left(\mathfrak{B}_{(}\left(\mathcal{H}_{0}\right)\right)$ the automorphism group of $\mathfrak{A}\left(\mathscr{K}_{0}\right)$, with the usual Borel structure [3].

Definition 1.2. A representation $\alpha$ of $(\stackrel{h}{h}, \Gamma, \mu)$ in $\mathfrak{H}_{0}$ is a Borel map $\alpha$ : R $\left.\right|_{E} \rightarrow \operatorname{Aut}\left(\mathscr{B}\left(\mathcal{H}_{0}\right)\right)$, where $\mu(\Gamma-E)=0$, satisfying $\alpha\left(\gamma, \gamma^{\prime}\right) \alpha\left(\gamma^{\prime}, \gamma^{\prime \prime}\right)=\alpha\left(\gamma^{\prime}, \gamma^{\prime \prime}\right)$ for all $\left(\gamma, \gamma^{\prime}\right)$ and $\left(\gamma^{\prime}, \gamma^{\prime \prime}\right)$ in $\left(\left.R\right|_{E}\right.$.

We shall use $(? h, \alpha)$ to denote a representation of $(h, \Gamma, \mu)$.

Given representations $($ ( $h, \alpha)$ and $($ ( h, $\beta)$ we may form their tensor product $\alpha \otimes \beta$, a representation in $\mathcal{H}_{0} \otimes \mathcal{H}_{0}$ by setting $(\alpha \otimes \beta)\left(\gamma, \gamma^{\prime}\right)=\alpha\left(\gamma, \gamma^{\prime}\right) \otimes \beta\left(\gamma, \gamma^{\prime}\right)$. The trivial representation is denoted $\iota$, so that $\iota\left(\gamma, \gamma^{\prime}\right)(x)=x$ for all $x \in \mathscr{B}\left(\mathcal{H}_{0}\right)$.

Definition 1.3. Representations $((h, \alpha)$ and $(? h, \beta)$ are

(i) conjugate, if there is a Borel map $\sigma: \Gamma \rightarrow \operatorname{Aut}\left(\mathcal{B}\left(\mathcal{K}_{0}\right)\right)$ with $\alpha\left(\gamma, \gamma^{\prime}\right)=$ $\sigma(\gamma) \beta\left(\gamma, \gamma^{\prime}\right) \sigma\left(\gamma^{\prime}\right)^{-1}$ on some i.r. and

(ii) quasi-conjugate if $\alpha \otimes \iota$ and $\beta \otimes \iota$ are conjugate.

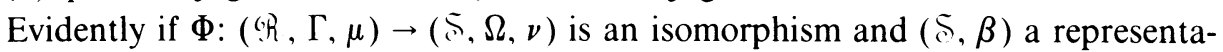
tion, and we define $\beta \cdot \Phi\left(\gamma, \gamma^{\prime}\right)=\beta\left(\Phi(\gamma), \Phi\left(\gamma^{\prime}\right)\right)$ then $\beta \cdot \Phi$ is a representation of $($ ( $R, \Gamma, \mu)$. This action of $\operatorname{Aut}(\stackrel{R}{*}, \mu)$ on the representations of $R$ clearly preserves conjugacy and quasi-conjugacy.

Definition 1.4. Representations ( $(R, \Gamma, \mu, \alpha)$ and $(\varsigma, \Omega, \nu, \beta)$ are

(i) equivalent if $\beta \cdot \Phi$ is conjugate to $\alpha$ for some isomorphism $\Phi$, and

(ii) quasi-equivalent if $\beta \otimes \imath$ is equivalent to $\alpha \otimes \imath$.

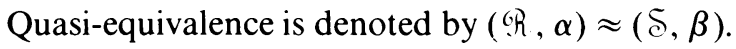

Definition 1.5. If ( $(R, \Gamma, \mu, \alpha)$ is a representation and $\Phi \in \operatorname{Aut}(\Re, \mu)$, we say $\Phi \in \operatorname{Aut}((h, \alpha)$ if $\alpha \cdot \Phi$ is quasi-conjugate to $\alpha$.

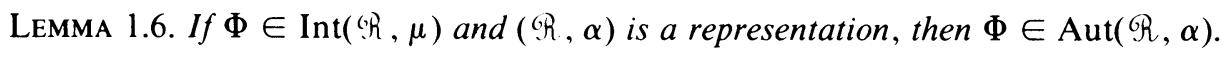

Proof. Choose a Borel map $a: \stackrel{G}{h} \rightarrow \mathcal{B}\left(\mathcal{H}_{0}\right)$ with unitary values and $\alpha\left(\gamma, \gamma^{\prime}\right)=$ Ad $a\left(\gamma, \gamma^{\prime}\right)$, and set $\sigma(\gamma)=\operatorname{Ad} a(\Phi(\gamma), \gamma)$. A straightforward computation now shows $\sigma(\gamma) \alpha\left(\gamma, \gamma^{\prime}\right) \sigma\left(\gamma^{\prime}\right)^{-1}=\alpha\left(\Phi(\gamma), \Phi\left(\gamma^{\prime}\right)\right)$.

Our primary interest is in quasi-equivalence classes of representations: as we shall see they are intrinsically related to maximal abelian subalgebras of von Neumann algebras, and thus to direct integrals of families of irreducible representations of $C^{*}$-algebras. The terminology is intentionally suggestive of that used in group representation theory. 
2. Algebras of sections. Let $\alpha$ be a representation of $(\mathcal{R}, \Gamma, \mu)$ in $\mathcal{H}_{0}$.

Definition 2.1. A section for $\alpha$ is an essentially bounded Borel map $\gamma \in E \rightarrow$ $x(\gamma) \in \mathfrak{B}\left(\mathcal{H}_{0}\right)$, where $\mu(\Gamma-E)=0$, with $\alpha\left(\gamma, \gamma^{\prime}\right)\left(x\left(\gamma^{\prime}\right)\right)=x(\gamma)$ for all $\left(\gamma, \gamma^{\prime}\right) \in$ $\left.\Re\right|_{E}$.

The set of all sections for $\alpha$ will be denoted $S(\alpha)$, which may evidently be considered as a von Neumann algebra on $L^{2}\left(\Gamma, \mu ; \mathcal{H}_{0}\right)$ via $(x \xi)(\gamma)=x(\gamma) \xi(\gamma)$ for $x \in S(\alpha), \xi \in L^{2}\left(\Gamma, \mu ; \mathcal{H}_{0}\right)$.

Proposition 2.2. If $(\mathcal{R}, \alpha)$ is a representation in $\mathcal{H}_{0}$, then $S(\alpha \otimes \iota)=S(\alpha) \otimes$ $\mathfrak{B}\left(\mathcal{H}_{0}\right)$.

Proof. Let $\mathfrak{B}=1 \otimes 1 \otimes \mathfrak{B}\left(\mathfrak{H}_{0}\right) \subseteq \mathfrak{B}\left(L^{2}\left(\Gamma, \mu ; \mathfrak{H}_{0}\right) \otimes \mathcal{H}_{0}\right) . \mathfrak{B}$ is type I, and $\mathfrak{B} \subseteq S(\alpha \otimes \imath)$ so that $S(\alpha \otimes \imath)=\left(S(\alpha \otimes \imath) \cap \mathfrak{B}^{\prime}\right) \otimes \mathfrak{B}$. But if $y \sim y(\gamma), y \in$ $S(\alpha \otimes \imath) \cap \mathfrak{B}^{\prime}$, we have $y(\gamma) x=x y(\gamma)$ for all $x \in \mathfrak{B}$. Hence $y(\gamma)=x(\gamma) \otimes 1 \in$ $\mathfrak{B}\left(\mathcal{H}_{0}\right) \otimes 1$. Since $\alpha\left(\gamma, \gamma^{\prime}\right)\left(x\left(\gamma^{\prime}\right)\right)=x(\gamma)$ on some i.r., $S(\alpha \otimes \imath) \cap \mathfrak{B}^{\prime}=S(\alpha) \otimes 1$, and $S(\alpha \otimes \iota)=S(\alpha) \otimes \mathfrak{B}\left(\mathcal{H}_{0}\right)$.

For every representation $(\Re, \alpha), S(\alpha)^{\prime}$ contains a copy of $L^{\infty}(\Gamma, \mu)$, acting on $L^{2}\left(\Gamma, \mu ; \mathcal{H}_{0}\right)$ in the obvious way; we denote this abelian subalgebra by $\mathcal{Q}(\Gamma)$ (or $\mathscr{Q}(\Gamma, \mu)$ if necessary). The pair $\left(S(\alpha)^{\prime}, \mathbb{Q}(\Gamma)\right)$ will be denoted $S^{\prime}(\Re, \alpha)$.

In general, it may well occur that $S(\alpha)=\mathrm{C} .1$, necessitating the following

Definition 2.3. A representation $(R, \alpha)$ is replete if $\mathscr{Q}(\Gamma) \subseteq S(\alpha)^{\prime}$ is a maximal abelian subalgebra.

Roughly speaking, $(\mathcal{G}, \alpha)$ is replete if for almost all $\gamma \in \Gamma,\{x(\gamma): x \in S(\alpha)\}$ generates $\mathfrak{B}\left(\mathcal{H}_{0}\right)$ (see $\S 3$ for further discussion).

Given von Neumann algebras $\Re, \Re$ with abelian (selfadjoint) subalgebras $\mathbb{Q}, \mathscr{R}$, respectively, we say $(\mathscr{R}, \mathbb{Q})$ is isomorphic with $(\mathscr{T}, \mathscr{B})$ if there is a normal isomorphism $\kappa: \mathfrak{N} \rightarrow \Re$ with $\kappa(Q)=\mathscr{B}$. In this case we write $(\mathfrak{R}, Q) \approx(\Re, \mathscr{R})$.

Proposition 2.4. Let $(\Re, \Gamma, \mu, \alpha)$ and $(\mathcal{S}, \Omega, \nu, \beta)$ be representations in $\mathcal{H}_{(0)}$. If $(\Re, \alpha) \approx(\delta, \beta)$ then $S^{\prime}(\Re, \alpha) \approx S^{\prime}(\mathcal{S}, \beta)$.

Proof. Let $\Phi:(\Re, \Gamma, \mu) \rightarrow(\mathcal{S}, \Omega, \nu)$ be an isomorphism, and $\gamma \rightarrow \sigma(\gamma) \in$ $\operatorname{Aut}\left(\mathscr{B}\left(\mathcal{H}_{0}\right)\right)$ be a Borel map with $\sigma(\Phi(\gamma))\left(\beta \cdot \Phi\left(\gamma, \gamma^{\prime}\right) \otimes \mathrm{id}\right) \sigma\left(\Phi\left(\gamma^{\prime}\right)\right)^{-1}=\alpha\left(\gamma, \gamma^{\prime}\right)$ $\otimes$ id on some i.r. of $\mathcal{R}$. Choose a Borel family of unitaries $\omega \in \Omega \rightarrow u(\omega) \in \mathfrak{B}\left(\mathcal{H}_{0}\right)$ with $\sigma(\omega)=\operatorname{Ad} u(\omega)$. From the above relation between $\alpha$ and $\beta$, if $\omega \rightarrow x(\omega) \in$ $\mathfrak{B}\left(\mathcal{H}_{0}\right)$ is a bounded Borel map then $x \in S(\beta \otimes \imath)$ if and only if $\tilde{x} \in S(\alpha \otimes \imath)$ where $\tilde{x}(\gamma)=u(\Phi(\gamma)) x(\Phi(\gamma)) u(\Phi(\gamma))^{*}$. Thus the unitary $u: L^{2}\left(\Omega, \nu ; \mathcal{H}_{0}\right) \rightarrow$ $L^{2}\left(\Gamma, \mu ; \mathcal{H}_{0}\right)$ given by $\left(u_{\Phi} \xi\right)(\gamma)=\rho(\gamma)^{-1 / 2} \xi(\Phi(\gamma))$, where $\rho(\gamma)=d \mu / d \nu \cdot \Phi(\gamma)$, implements an isomorphism of $S(\beta \otimes \imath)=S(\beta) \otimes \mathfrak{B}\left(\mathcal{H}_{0}\right)$ with $S(\alpha) \otimes \mathfrak{B}\left(\mathcal{H}_{0}\right)$. The conclusion is immediate.

The converse to 2.4. is false.

EXAMPLE 2.5 . Let $(\Gamma, \mu)=(\Omega, \nu)$, let $\Re$ be the equivalence relation generated by an ergodic action of some countable group on $(\Gamma, \mu)$, let $\delta=\Gamma \times \Gamma$, and let $\alpha=\beta=\imath$ on the appropriate domain. By ergodicity, $S(\alpha)=1 \otimes \mathfrak{B}\left(\mathcal{H}_{0}\right)=S(\beta)$, but in general, $(\Re, \alpha)$ is not quasi-equivalent with $(\delta, \beta)$.

Conditions under which the converse to 2.4 holds will be established in 3.6. 
3. Takesaki's equivalence relation. Takesaki, in [14], has shown how to associate to each pair $(\mathfrak{N}, \mathbb{Q})$ consisting of a von Neumann algebra $\Re$ with separable predual, with a maximal abelian subalgebra $\mathscr{Q} \subseteq \mathfrak{N}$, an analytic equivalence relation $\Re(\mathscr{N}, \mathscr{Q})$ on a standard measure space. Briefly, we may suppose $\mathfrak{N}$ is represented on a separable Hilbert space $\mathcal{H}$, and choose a separable $C^{*}$-algebra $A \subseteq \Re^{\prime}$, $\sigma$-weakly dense in $\mathfrak{T}^{\prime}$. Decomposition of the identity representation $\iota_{A}$ of $A$ over $Q$, $\iota_{A}=\int_{\Gamma}^{\oplus} \pi_{\gamma}^{A} d \mu(\gamma)$, gives rise to an analytic equivalence relation $\Re(\Re, Q, A)$ on $\Gamma$ via $\left(\gamma, \gamma^{\prime}\right) \in \mathcal{R}(\mathfrak{N}, \mathbb{Q}, A)$ if and only if the irreducible representations $\pi_{\gamma}^{A}$ and $\pi_{\gamma^{\prime}}^{A}$ are unitarily equivalant. By [14], different choices for $A$ yield isomorphic equivalence relations, as do different realizations of $(\mathfrak{N}, \mathcal{Q})$ on Hilbert space. We denote this equivalence relation by $\mathcal{R}(\mathfrak{R}, \mathbb{Q})$. More however is involved; with the above notation, we may choose a realization of $(\mathscr{R}, \mathscr{Q})$ so that in the decomposition of $\iota_{A}$ over $\mathcal{Q}$, we may assume each of the representations $\pi_{\gamma}^{A}$ acts on a fixed Hilbert space $\mathcal{H}_{0}$. Also, if for each $\left(\gamma, \gamma^{\prime}\right) \in \mathscr{R}(\mathfrak{N}, \mathbb{Q}, A)$ we let $\alpha^{A}\left(\gamma, \gamma^{\prime}\right)$ be the unique automorphism of $\mathscr{B}\left(\mathcal{H}_{0}\right)$ with $\alpha^{A}\left(\gamma, \gamma^{\prime}\right) \circ \pi_{\gamma^{\prime}}^{A}=\pi_{\gamma}^{A}$, then $\alpha^{A}$ is a representation of $\Re(\Re, Q, A)$. The proof which shows that the isomorphism class of $R(\Re, \mathcal{Q}, A)$ depends only on the isomorphism class of $(\mathcal{N}, \mathbb{Q})$ simultaneously shows that the quasi-equivalence class of $\alpha^{A}$ depends only on the pair ( $\mathscr{R}, \mathbb{Q}$ ). Since the operator fields $\left\{\pi_{\gamma}^{A}(x) ; \gamma \in \Gamma\right\}, x \in A$, lie in $S\left(\alpha^{A}\right), \alpha^{A}$ is replete. We denote by $\mathcal{T}(\mathcal{T}, \mathbb{Q})=$ $(\mathscr{R}(\mathfrak{T}, \mathbb{Q}), \alpha(\mathfrak{T}, \mathbb{Q}))$ the representation of $\mathcal{R}(\mathfrak{T}, \mathbb{Q})$ obtained as above.

Proposition 3.1. If $(\Re, \mathscr{Q}) \approx(\Re, \Re)$ then $\mathscr{T}(\Re, \mathscr{Q}) \approx \mathcal{T}(\Re, \mathscr{B})$.

Proof. See [14] for this result, stated in different language.

The converse to 3.1. fails also, as is seen by easy examples.

Note also that $S^{\prime}(\mathscr{T}(\mathfrak{N}, \mathbb{Q})) \approx(\mathscr{T}, \mathbb{Q})$ may also occur. For example if $\mathbb{Q} \subseteq \Re$ is simple, so $\mathscr{R}(\mathfrak{R}, \mathbb{Q})=\{(\gamma, \gamma): \gamma \in \Gamma\}$, then $S^{\prime}(\mathcal{T}(\mathfrak{R}, \mathbb{Q}))=\left(L^{\infty}(\Gamma, \mu) \otimes 1\right.$, $\left.L^{\infty}(\Gamma, \mu) \otimes 1\right)$. (See [13] for examples of this phenomenon.) As noted in 2.5 $\mathcal{T}\left(S^{\prime}(\Re, \alpha)\right) \approx(\Re, \alpha)$ may also occur; there $\mathcal{T}\left(S^{\prime}(\Re, \iota)\right)=(\mathcal{S}, \iota)$.

Given a pair $(\mathscr{R}, \mathbb{Q})$ with $Q$ maximal abelian, we may construct a chain of such pairs $\left(\Re_{j}, \mathscr{Q}\right), j \geqslant 0$, by setting $\Re_{0}=\Re$, and, inductively $\left(\Re_{j+1}, \mathscr{Q}\right)=$ $S^{\prime}\left(\mathcal{T}\left(\Re_{j}, \mathscr{Q}\right)\right)$. Equally, given a replete representation $(\Re, \alpha)$, set $\Re_{0}=\mathscr{R}, \alpha_{0}=\alpha$ and $\left(\Re_{j+1}, \alpha_{j+1}\right)=\mathcal{J}\left(S^{\prime}\left(\Re_{j}, \alpha_{j}\right)\right), j \geqslant 0$, and each $\left(\Re_{j}, \alpha_{j}\right)$ is again a replete representation. These chains both stabilize at $j=1$, as seen in

Proposition 3.2. With the above notation

(i) $\Re_{1} \subseteq \Re ; \Re_{1} \supseteq \Re$ and $\alpha_{1}=\alpha$ on $R$.

(ii) $\mathscr{T}(\mathscr{T}, \mathbb{Q})=\mathscr{T}\left(\Re_{1}, \mathscr{Q}\right)$.

(iii) $S^{\prime}(\Re, \alpha)=S^{\prime}\left(\Re_{1}, \alpha_{1}\right)$.

Proof. (i) Let ( $\Re, Q$ ) be given, and $A \subseteq \Re^{\prime}$ be as usual; let $\iota_{A}=\int_{\Gamma}^{\oplus} \pi_{\gamma}^{A} d \mu(\gamma)$ be the decomposition over $Q$. Since $\alpha^{A}\left(\gamma, \gamma^{\prime}\right) \pi_{\gamma^{\prime}}^{A}(x)=\pi_{\gamma}^{A}(x)$ for $x \in A, A \subseteq S\left(\alpha^{A}\right)$ and $\mathscr{N}_{1}=S\left(\alpha^{A}\right)^{\prime} \subseteq \Re$.

If ( $R, \alpha)$ is given and $A \subseteq S(\alpha)$ is a $\sigma$-weakly dense separable $C^{*}$-subalgebra with decomposition $\iota_{A}=\int_{\Gamma}^{\oplus} \pi_{\gamma}^{A} d \mu(\gamma)$, we have $\pi_{\gamma}^{A}(x)=x(\gamma)$ a.e., so the second assertion of (i) is clear. 


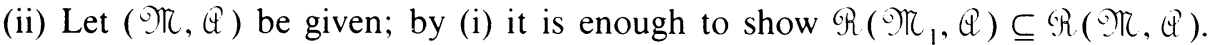
Let $A \subseteq \mathcal{N}^{\prime}, A_{1} \subseteq \mathcal{M}_{1}^{\prime}$ be $\sigma$-weakly dense separable $C^{*}$-subalgebras; since $\mathfrak{N}_{1} \subseteq$ ON, we may assume $A_{1} \supseteq A$. Hence, in the decompositions $\iota_{A}=\int_{\Gamma}^{\oplus} \pi_{\gamma}^{A} d \mu(\gamma)$, $\iota_{A_{1}}=\int_{\Gamma}^{\oplus} \pi_{\gamma}^{A_{1}} d \mu(\gamma)$, we may assume $\pi_{\gamma}^{A_{1}}$ extends $\pi_{\gamma}^{A} \mu$-a.e. on $\Gamma$, and the inclusion $\mathscr{R}\left(\mathscr{N}_{1}, \mathscr{U}\right) \subseteq \Re(\mathscr{N}, \mathbb{Q})$ is apparent.

(iii) If $(\mathcal{R}, \alpha)$ is given, then since $\alpha_{1}$ extends $\alpha$ by (i), $S\left(\alpha_{1}\right) \subseteq S(\alpha)$ and $S(\alpha)^{\prime} \subseteq$ $S\left(\alpha_{1}\right)^{\prime}$. Conversely applying (1) to $S^{\prime}(\Re, \alpha)$ we see $S\left(\alpha_{1}\right)^{\prime} \subseteq S(\alpha)^{\prime}$ as required.

Definition 3.3. (i) A replete representation $(\Re, \alpha)$ is complete if $T\left(S^{\prime}(\Re, \alpha)\right)=$ $(\mathscr{R}, \alpha)$.

(ii) A von Neumann algebra, maximal abelian subalgebra pair ( $\mathcal{R},(\vec{Q})$ is complete if $S^{\prime}(\mathscr{T}(\mathcal{O}, \mathbb{Q}))=(\mathfrak{O}, \mathbb{Q})$.

There are evidently dual notions; by 3.2 pairs ( $(R, \alpha)$ and ( OR, (Q) may be "completed" (although in the case of von Neumann algebras this involves taking a subalgebra of $\mathfrak{N})$. As we shall see, if $\mathscr{Q} \subseteq \mathfrak{N}$ is regular and maximal abelian, $(\mathfrak{N}, \mathbb{Q})$ is complete; however, completeness is a more general notion than regularity. The completion of a pair $(6 R, \alpha)$ should probably be thought of as a sort of "maximal extension" of $(\because R, \alpha)$; an internal description of the completion of $(? R, \alpha)$ would be highly desirable, but this author is unable to find such a description.

The preceding discussion also indicates how to construct pairs ( $9 \pi, \mathrm{C}^{3}$ ) with specified $\Re(\Re, Q)$. Let $B$ be a separable $C^{*}$-algebra, $\left\{\pi_{\gamma}: \gamma \in \Gamma\right\}$ a measurable family of irreducible representations of $B$ on $\mathcal{K}_{0}$, and let $\mu$ be a measure on $\Gamma$ so that $\pi=\int_{\Gamma}^{\oplus} \pi_{\gamma} d \mu(\gamma)$ is faithful. Let $\delta=\left\{\left(\gamma, \gamma^{\prime}\right): \pi_{\gamma}\right.$ and $\pi_{\gamma^{\prime}}$ are unitarily equivalent $\}$, and let $\beta$ be the representation of $\$$ with $\beta\left(\gamma, \gamma^{\prime}\right) \cdot \pi_{\gamma^{\prime}}=\pi_{\gamma}$ for $\left(\gamma, \gamma^{\prime}\right) \in \widehat{s}$.

Proposition 3.4. With the above notation, $\left.\stackrel{G(R}{(\pi}(B)^{\prime}, L^{\infty}(\Gamma, \mu) \otimes 1\right)=(\varsigma, \beta)$.

The proof follows as in 3.3(ii). Note the choice of $\mu$ is almost completely arbitrary.

We now turn to automorphisms. Let $(6 R, \alpha)$ be a replete representation. We adopt the following notations: $\mathcal{C}(\stackrel{R}{R}, \alpha)$ is the group of all Borel maps $\gamma \rightarrow u(\gamma)$, with $u(\gamma)$ unitary a.e., such that $\operatorname{Ad} u(\gamma) \cdot \tilde{\alpha}\left(\gamma, \gamma^{\prime}\right) \cdot \operatorname{Ad} u\left(\gamma^{\prime}\right)^{-1}=\tilde{\alpha}\left(\gamma, \gamma^{\prime}\right)$ on some i.r., where $\tilde{\alpha}\left(\gamma, \gamma^{\prime}\right)=\alpha\left(\gamma, \gamma^{\prime}\right) \otimes$ id and we identify maps if they agree a.e. $\mathcal{E}_{0}(? h, \alpha)$ is the subgroup of $e(\Re, \alpha)$ of maps with scalar values. Aut $(R, \alpha)$ is as in 1.5 and

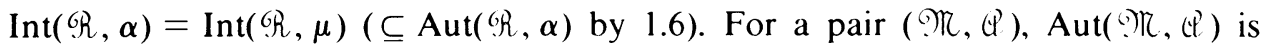

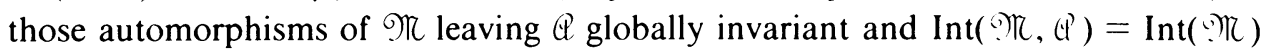
$\cap \operatorname{Aut}(\mathfrak{T}, Q)$.

Proposition 3.5. If $(G R, \alpha)$ is complete, there are exact sequences

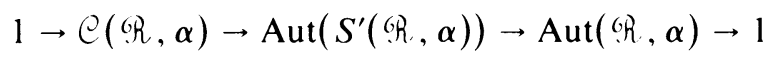

and

$$
1 \rightarrow e_{0}(\Re, \alpha) \rightarrow \operatorname{Int}\left(S^{\prime}(G R, \alpha)\right) \rightarrow \operatorname{Int}(\Re R, \alpha) \rightarrow 1 .
$$

Proof. Let $\gamma \rightarrow u(\gamma)$ be in $\mathcal{C}(\Re, \alpha)$, and let $A \subseteq S(\tilde{\alpha})$ be as usual. Define a unitary $u$ on $L^{2}\left(\Gamma, \mu ; \mathcal{H}_{0} \otimes \mathscr{H}_{0}\right)$ by $(u \xi)(\gamma)=u(\gamma) \xi(\gamma)$. If $x \sim x(\gamma) \in$ $L^{\infty}\left(\Gamma, \mu ; \mathfrak{B}\left(\mathcal{H}_{0} \otimes \mathcal{H}_{0}\right)\right)$, then $u x u^{*} \sim \operatorname{Ad} u(\gamma)(x(\gamma))$. Hence if $x \in S(\tilde{\alpha})$,

$$
\begin{aligned}
\tilde{\alpha}\left(\gamma, \gamma^{\prime}\right)\left(\left(u x u^{*}\right)\left(\gamma^{\prime}\right)\right) & =\tilde{\alpha}\left(\gamma, \gamma^{\prime}\right) \cdot \operatorname{Ad} u\left(\gamma^{\prime}\right)(x(\gamma)) \\
& =\operatorname{Ad} u(\gamma) \tilde{\alpha}\left(\gamma, \gamma^{\prime}\right)\left(x\left(\gamma^{\prime}\right)\right)=\operatorname{Ad} u(\gamma) x(\gamma)
\end{aligned}
$$


on some i.r., and $u S(\tilde{\alpha}) u^{*} \subseteq S(\tilde{\alpha})$; thus $\operatorname{Ad} u$ defines an automorphism $\theta_{u}$ of $S(\alpha)^{\prime} \otimes 1$, and hence of $S(\alpha)^{\prime}$.

Clearly, if $u \in \mathcal{E}_{0}(? R, \alpha), \theta_{u}$ is inner. Conversely, if $\theta_{u}$ is inner then, since $\theta_{u}(a)=a$ for all $a \in \mathcal{C}^{\prime}(\Gamma)$ and $\alpha$ is replete, $u$ has scalar values.

Let $\varphi \in \operatorname{Aut}\left(S^{\prime}(h, \alpha)\right)$ be arbitrary; passing to $S^{\prime}(\Re, \alpha) \otimes 1$, we may assume $\varphi=\operatorname{Ad} v$ for some unitary $v$. By [12], since $\varphi\left(\epsilon^{\prime}(\Gamma)\right)=\epsilon^{\top}(\Gamma)$, we have $\varphi(f)=f \cdot \Phi^{-1}$ for some invertible Borel map $\Phi: \Gamma \rightarrow \Gamma$ with $\mu \cdot \Phi$ equivalent to $\mu$, for each $f \in L^{\infty}(\Gamma, \mu)$. We show $\Phi \in \operatorname{Aut}(\Re, \alpha)$. By [13], $v$ is of the form $(v \xi)(\gamma)=$ $v(\gamma) \xi\left(\Phi^{-1}(\gamma)\right) \rho_{\Phi}(\gamma)^{1 / 2}$ where $\rho_{\Phi}(\gamma)=d \mu / d \mu \cdot \Phi^{-1}(\gamma)$ and $v(\gamma)$ is unitary. Since $\varphi \in \operatorname{Aut}\left(S(\alpha)^{\prime}\right), v S(\tilde{\alpha}) v^{*}=S(\tilde{\alpha})$. Thus if $A \subseteq S(\tilde{\alpha})$ is as usual, we may assume $v A v^{*}=A$. Now for $x \sim x(\gamma) \in L^{\infty}\left(\Gamma, \mu\right.$; $\left.B\left(\mathcal{H}_{0} \otimes \mathbb{K}_{0}\right)\right)$ we have

$$
v x v^{*} \sim v(\gamma) x\left(\Phi^{-1}(\gamma)\right) v(\gamma)^{*} .
$$

Hence for $x \in A, \pi_{\gamma}^{A}\left(v x v^{*}\right)=\operatorname{Ad} v(\gamma) \pi_{\Phi^{-1}(\gamma)}^{A}(x)$ and so, $\pi_{\gamma}^{A} \cdot \operatorname{Ad} v=\operatorname{Ad} v(\gamma) \pi_{\Phi^{-1}(\gamma)}^{A}$ $\mu$-a.e. Thus, since $((h, \alpha)$ (and hence $(\mathcal{h}, \tilde{\alpha})$ also) is complete, we have $\tilde{\alpha}\left(\gamma, \gamma^{\prime}\right) \cdot \pi_{\gamma^{\prime}}{ }^{A}=\pi_{\gamma}^{A}$ on some i.r. and hence $\operatorname{Ad} v(\gamma)^{*} \cdot \tilde{\alpha}\left(\gamma, \gamma^{\prime}\right) \cdot \operatorname{Ad} v\left(\gamma^{\prime}\right)$ implements an isomorphism of $\pi_{\Phi^{-1}\left(\gamma^{\prime}\right)}^{A}$ with $\pi_{\Phi^{-1}(\gamma)}^{A}$. Thus $\operatorname{Ad} v(\gamma)^{*} \cdot \tilde{\alpha}\left(\gamma, \gamma^{\prime}\right) \cdot \operatorname{Ad} v\left(\gamma^{\prime}\right)=$ $\tilde{\alpha}\left(\Phi^{-1}(\gamma), \Phi^{-1}\left(\gamma^{\prime}\right)\right)$ and $\Phi \in \operatorname{Aut}(\stackrel{R}{R}, \alpha)$.

If $\varphi$ is inner, $v x v^{*}=x$ for all $x \in A$, and $\pi_{\gamma}^{A}$ is unitarily equivalent with $\pi_{\Phi^{-1}(\gamma)}^{A}$ for almost all $\gamma$.

We now show the maps $\varphi \in \operatorname{Aut}\left(S^{\prime}(\Re, \alpha)\right) \rightarrow \Phi \in \operatorname{Aut}(\Re, \alpha)$ and $\operatorname{Int}\left(S^{\prime}(\Re, \alpha)\right)$

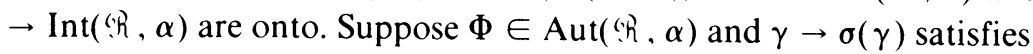

$$
\sigma(\gamma) \tilde{\alpha}\left(\gamma, \gamma^{\prime}\right) \sigma\left(\gamma^{\prime}\right)^{-1}=\tilde{\alpha}\left(\Phi^{-1}(\gamma), \Phi^{-1}\left(\gamma^{\prime}\right)\right) .
$$

Choose a Borel family $\gamma \rightarrow v(\gamma)$ of unitaries with $\sigma(\gamma)=\operatorname{Ad} v(\gamma)^{*},(\sigma(\gamma) \in$ Aut $\left(\mathcal{H}^{(}\left(\mathfrak{K}_{0}\right)\right)$ remember), and define a unitary $v$ on $L^{2}\left(\Gamma, \mu ; \mathcal{H}_{0} \otimes \mathscr{H}_{0}\right)$ by $(v \xi)(\gamma)$ $=v(\gamma) \xi\left(\Phi^{-1}(\gamma)\right) \rho_{\Phi}(\gamma)^{1 / 2}$, as above; a routine computation shows that $\operatorname{Ad} v(S(\tilde{\alpha}))$ $=S(\tilde{\alpha})$ and that $\operatorname{Ad} v(f)=f \cdot \Phi^{-1}$ for $f \in \mathcal{Q}^{\prime}(\Gamma)$. Note that if $\Phi$ is inner, then choosing a Borel map $\left(\gamma, \gamma^{\prime}\right) \in\left(h \rightarrow a\left(\gamma, \gamma^{\prime}\right)\right.$, with $a\left(\gamma, \gamma^{\prime}\right)$ unitary on $\mathcal{H}_{0} \otimes \mathcal{H}_{0}$ and $\tilde{\alpha}\left(\gamma, \gamma^{\prime}\right)=\operatorname{Ad} a\left(\gamma, \gamma^{\prime}\right)$ (as in 1.6), we may choose $v(\gamma)$ in the above discussion with $v(\gamma)=a\left(\Phi^{-1}(\gamma), \gamma\right)^{*}$. Now for $x \in S(\tilde{\alpha})$ we have

$$
\begin{aligned}
v x v^{*} & \sim v(\gamma) x\left(\Phi^{-1}(\gamma)\right) v(\gamma)^{*}=\operatorname{Ad} a\left(\Phi^{-1}(\gamma), \gamma\right)^{*}\left(x\left(\Phi^{-1}(\gamma)\right)\right. \\
& =\alpha\left(\gamma, \Phi^{-1}(\gamma)\right)\left(x\left(\Phi^{-1}(\gamma)\right)=x(\gamma),\right.
\end{aligned}
$$

so that $v x v^{*}=x$ and Ad $v$ defines an inner automorphism of $S(\alpha)^{*}$.

The remaining step in the proofs is trivial, and left to the reader.

Corollary 3.6. If $($ ( $R, \alpha)$ and $(\varsigma, \beta)$ are complete, then $S^{\prime}(\Re, \alpha) \approx S^{\prime}(\mathcal{S}, \beta)$ if

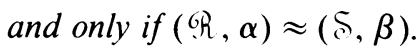

The proof is immediate from 2.4 and the considerations of 3.5.

There is an evident dual proposition concerning $\operatorname{Aut}(\mathscr{T}(\mathscr{T}, \mathbb{Q}))$, and a corollary dual to 3.6 for complete pairs $(\mathscr{R}, \mathscr{Q})$ and $(\mathcal{X}, \mathscr{B})$ which we omit.

We return to an example.

EXAmPLE 3.7. Let $\Gamma=\Pi Z_{2}$, and let $\pi_{\gamma}, \gamma \in \Gamma$, be a Borel family of irreducible representations of the $2 \times 2$-U.H.F. algebra such that $\pi_{\gamma}$ is unitarily equivalent to $\pi_{\gamma^{\prime}}$ 
if and only if $\gamma-\gamma^{\prime} \in \amalg Z_{2}$ (such families clearly exist). Let $\mu$ be a Borel measure on $\Gamma$ such that $\mu$ is singular with respect to $\mu_{g}$ for all $g \in G=\amalg Z_{2}$, where $\mu_{g}(E)=$ $\mu(E+g)$. From 3.4, we obtain a pair $(\mathfrak{R}, \mathbb{Q})$ with $\mathscr{T}(\mathscr{T}, \mathbb{Q})=(\mathcal{S}, \Gamma, \mu, \beta)$ where $\delta$ is generated by translation by $G$ on $\Gamma$. Since each $\Phi \in \operatorname{Int}(\delta, \mu)$ is in the full group of $G$, i.e. locally translation by some element of $G$ (see $[3,4,5]$ ), and none of these transformations preserve the class of $\mu$ (except the identity), Int $(\mathfrak{O}, \mathscr{Q})=\bigodot_{0}(\Re, \alpha)$, and $Q \subseteq \mathfrak{K}$ is singular in the sense of [14]. However, $Q \subseteq \mathfrak{K}$ is not simple in the sense of [14], thus answering a question raised in [14].

4. Comparison of $(\Re, Q)$ with $\Re(\mathscr{R}, Q)$ and completeness. We fix $Q \subseteq \Re$, a maximal abelian subalgebra, and relate properties of $(\mathfrak{R}, \mathbb{Q})$ to those of $\mathscr{T}(\mathfrak{T}, \mathbb{Q})$. We shall say that an analytic equivalence relation $(\Re, \Gamma, \mu)$ is countable nonsingular if $\Re(\gamma)$ is countable for almost all $\gamma \in \Gamma$, and if whenever $E \subseteq \Gamma$ is Borel and null, $\mu(\Re(E))=0$. Feldman and Moore $[4,5]$ have axiomatized these equivalence relations; they are precisely those which may be generated by nonsingular actions of countable groups on $(\Gamma, \mu)$.

THEOREM 4.1. $Q \subseteq \mathfrak{T}$ is regular if and only if $(\mathfrak{R}, Q) \approx S^{\prime}(\Re, \alpha)$ for some replete representation of some countable nonsingular equivalence relation $(\Re, \Gamma, \mu)$.

Proof. Suppose $\mathscr{Q} \subseteq \mathcal{N}$ is regular, and let $\left\{u_{g}: g \in G\right\}$ be a countable group of unitaries in $\mathscr{N}$, normalizing $\mathcal{Q}$, and, together with $\mathcal{Q}$, generating $\mathfrak{N}$. Denote the point realization of $G$ on $\mathbb{Q}=L^{\infty}(\Gamma, \mu)$ by $(\gamma, g) \in \Gamma \times G \rightarrow \gamma g \in \Gamma$; each transformation $\gamma \rightarrow \gamma g$ preserves the class of $\mu$, and we write $\rho(\gamma, g)=d \mu(\cdot g) / d \mu(\gamma)$, where $\mu(E g)=\mu(\cdot g)(E)$, for $E \subseteq \Gamma$, Borel (see [12]).

If $\mathscr{N}$ acts on $\mathcal{H}$, we may suppose that in the decomposition $\mathcal{H}=\int_{\Gamma}^{\oplus} \mathcal{H}(\gamma) d \mu(\gamma)$ over $Q$ that $\mathcal{H}(\gamma)=\mathcal{H}_{0}$ a.e., and that each $u_{g}$ is expressed as $\left(u_{g} \xi\right)(\gamma g)=$ $\rho(\gamma, g)^{-1 / 2} u_{g}(\gamma) \xi(\gamma)$, where $u_{g}(\gamma)$ is unitary on $\mathcal{H}_{0}[13]$. Note that if $E_{g, h}=\{\gamma \in \Gamma$ : $\gamma g=\gamma h$ \} corresponds to the projection $e_{g, h} \in \mathbb{Q}, u_{g}^{*} u_{h} e_{g, h}$ commutes with $\mathbb{Q}$, which is maximal abelian, so that $u_{h} e_{g, h}=z u_{g} e_{g, h}$ for some $z \in \mathbb{Q}$. Thus $u_{g}(\gamma)=z(\gamma) u_{h}(\gamma)$ on $E_{g, h}$, for some $z(\gamma) \in \mathbf{T}$, and there is a well-defined representation $\alpha$ of the equivalence relation $\Re$ generated by the action of $G$ on $(\Gamma, \mu)$ given by $\alpha(\gamma g, \gamma)=$ Ad $u_{g}(\gamma)$.

We claim $(\Re, \mathscr{Q})=S^{\prime}(\Re, \alpha)$. Note $x \in S(\alpha)$ if and only if for each $g \in G$, $u_{g}(\gamma) x(\gamma)=x(\gamma g) u_{g}(\gamma)$ a.e., and thus if and only if $u_{g} x=x u_{g}\left(\right.$ since $\left(u_{g} x \xi\right)(\gamma g)=$ $\rho(\gamma, g)^{-1 / 2} u_{g}(\gamma) x(\gamma)$, and similarly for $\left.x u_{g}\right)$. Thus $S(\alpha)=L^{\infty}\left(\Gamma, \mu ; \mathfrak{B}\left(\mathcal{H}_{0}\right)\right) \cap$ $\left\{u_{g}\right\}^{\prime}$, and so $S(\alpha)^{\prime}$ is generated by $L^{\infty}(\Gamma, \mu) \otimes 1$ and $\left\{u_{g}: g \in G\right\}$.

Conversely, if $(\Re, \mathbb{Q})=S^{\prime}(\Re, \alpha)$ with $\Re$ countable nonsingular, we may choose a countable group $G$ and a nonsingular action of $G$ on $(\Gamma, \mu)$ which generates $R$. Also, choosing a Borel map $a:\left(\gamma, \gamma^{\prime}\right) \in \Re \rightarrow a\left(\gamma, \gamma^{\prime}\right) \in \mathfrak{B}\left(\mathcal{H}_{0}\right)$ with $a\left(\gamma, \gamma^{\prime}\right)$ unitary and $\alpha\left(\gamma, \gamma^{\prime}\right)=\operatorname{Ad} a\left(\gamma, \gamma^{\prime}\right)$ on some i.r. of $R$, we may define unitaries $u_{g}$ on $L^{2}\left(\Gamma, \mu ; \mathcal{H}_{0}\right)$ by $\left(u_{g} \xi\right)\left(\gamma_{g}\right)=a(\gamma g, \gamma) \rho(\gamma, g)^{-1 / 2} \xi(\gamma)$. The preceding argument shows that $x \in S(\alpha)$ if and only if $x \in \mathbb{Q}^{\prime} \cap\left\{u_{g}\right\}^{\prime}$ so that $\mathscr{Q} \subseteq S(\alpha)^{\prime}$ is regular, since $u_{g} Q u_{g}^{*}=Q$.

Thus if $\mathcal{Q} \subseteq \mathcal{M}$ is regular, $\mathcal{T}(\mathfrak{N}, \mathcal{Q})$ is the completion of a representation $(\mathscr{R}, \alpha)$ with $R$ countable nonsingular. Also 
COROLlaRy 4.2. If $\Theta \subseteq \mathfrak{M}$ is regular maximal abelian, then $(\mathcal{M}, \mathbb{U}$ ) is complete.

As promised we now show that completeness properly generalizes regularity.

EXAMPLE 4.3. Let $\left\{\pi_{\gamma}: \gamma \in \Pi Z_{2}\right\}$ be the representations of Example 3.7, and let $(\mathscr{R}, \mathscr{G})$ be the naturally associated equivalence relation and representation, so that $\beta\left(\gamma, \gamma^{\prime}\right) \cdot \pi_{\gamma^{\prime}}=\pi_{\gamma}$. Let $(\mathcal{N}, \mathscr{Q})=S^{\prime}(\Re, \beta)$. If $\mathcal{O} \pi$ is the algebra of 2.7 , then since $\mathfrak{M} \supset \mathfrak{X} \supset \mathbb{Q}$ and $\mathbb{Q} \subseteq \mathcal{N}$ is singular, $\mathcal{Q}$ is singular in $\mathcal{N}$. On the other hand $(\mathcal{N}, \mathbb{Q})$ is by definition complete.

EXAMPLE 4.4. Let

$$
G=\left\{\left(\begin{array}{ccc}
1, & m, & t \\
0, & 1, & n \\
0, & 0, & 1
\end{array}\right): m, n \in Z, t \in \mathbf{R}\right\}, \quad g_{n}=\left(\begin{array}{ccc}
1, & 0, & 0 \\
0, & 1, & n \\
0, & 0, & 1
\end{array}\right)
$$

and $H \subseteq G$ be the subgroup given by $n=0$. (So $H$ is isomorphic with $Z \times \mathbf{R}$.) Let $\rho_{\varphi}$ be the representation of $H, \rho_{\varphi}(m, t)=\exp i(t+m \varphi)$ (this representation is denoted $\chi_{1, \varphi}$ in [9, p. 296]). Set $\pi_{\varphi}=\operatorname{Ind}_{H}^{G} \rho_{\varphi}$ and $\pi=\int_{\mathbf{R}}^{\oplus} \pi_{\varphi} d \varphi$. It is easily checked that (after taking the Fourier transform), $\pi_{\varphi}$ and $\pi$ may be realized via

$$
\left.\begin{array}{l}
\left(\pi\left(g_{n}\right) \xi\right)(\varphi, s)=e^{i n s} \xi(\varphi, s) \\
(\pi(m, r) \xi)(\varphi, s)=e^{i(r+m \varphi)} \xi(\varphi, s+m)
\end{array}\right\}, \quad \xi \in L^{2}(\mathbf{R} \times[0,2 \pi)),
$$

and

$$
\left.\begin{array}{l}
\left(\pi_{\varphi}\left(g_{n}\right) \xi\right)(s)=e^{i n s} \xi(s) \\
\left(\pi_{\varphi}(m, t) \xi\right)(s)=e^{i(t+m \varphi)} \xi(s+m)
\end{array}\right\}, \quad \xi \in L^{2}([0,2 \pi)) .
$$

The $\pi_{\varphi}$ are irreducible, and $\pi_{\varphi}$ is unitarily equivalent with $\pi_{\varphi^{\prime}}$ if and only if $\varphi-\varphi^{\prime}=2 \pi k+l$ for some $k, l \in Z$. Also if $\left(u\left(\varphi^{\prime}, \varphi\right) \xi\right)(s)=e^{i l s} \xi(s) \quad(\xi \in$ $\left.L^{2}([0,2 \pi))\right)$ where $\varphi^{\prime}=\varphi-2 \pi h-l$, then $\operatorname{Ad} u\left(\varphi^{\prime}, \varphi\right) \cdot \pi_{\varphi}=\pi_{\varphi^{\prime}}$.

Thus if $\Re_{1}=\pi(G)^{\prime}, \Re\left(\Re_{1}, L^{\infty}(\mathbf{R}) \otimes 1\right)=\mathscr{R}$ is generated by the action of $Z \times Z$ on $\mathbf{R}$ given by $\varphi \cdot(k, l)=\varphi-2 \pi k-l(k, l \in Z)$. Thus if $\Re \subseteq \Re_{1}$ is the von Neumann subalgebra generated by the normalizer of $L^{\infty}(\mathbf{R}) \otimes 1$ in $\Re_{1}, \Re\left(\Re, L^{\infty}(\mathbf{R}) \otimes 1\right)=\Re$ also. But (see proof of 4.1 ), $\Re$ is generated on $L^{2}(\mathbf{R} \times[0,2 \pi))$ by operators of the form $a \otimes 1, a \in L^{\infty}(\mathbf{R})$ and $u_{n, l}=v_{n, l} \otimes \chi_{l}$ where $\left(v_{k, l} \xi\right)(\varphi)=\xi(\varphi-2 \pi k-l)$ (for $\left.\xi \in L^{2}(\mathbf{R})\right)$ and $\left(\chi_{l} \eta\right)(s)=e^{i l s} \eta(s)$ (for $\left.\eta \in L^{2}([0,2 \pi))\right)$.

Note that if there is a conditional expectation from $\mathfrak{T}$ to $\mathcal{Q}$, then by Feldman and Moore [5], $\Re$ is the crossed product of $L^{\infty}(\mathbf{R})$ by the above (free, ergodic) action of $Z \times Z$. (Note $R$ is hyperfinite as an equivalence relation by [3], and so all problems of second cohomology for $R$ disappear by [4].) With the observation that the representations $(k, l) \rightarrow u_{k, l}$ and $(k, l) \rightarrow u_{k, l} \otimes \chi_{k}$ of $Z \times Z$ are not quasi-equivalent, the desired result follows from

Proposition 4.5. Let $G$ be countable group acting freely on $(\Gamma, \mu)$ and $\pi$ be a representation of $G$ on $\mathcal{H}$. Let $\mathscr{N}$ be the algebra on $L^{2}(\Gamma, \mu) \otimes \mathfrak{H}$ generated by the operators $a \otimes 1, a \in L^{\infty}(\Gamma, \mu)$ and $u_{g}=v_{g} \otimes \pi(g)$ where $\left(v_{g} \xi(\gamma)\right)=\rho(\gamma, g)^{1 / 2} \xi(\gamma g)$ 
on $L^{2}(\Gamma, \mu)$. Suppose $L^{\infty}(\Gamma, \mu) \otimes 1$ is maximal abelian in $\Re$. Then if there is a

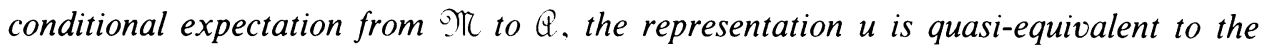
regular representation of $G$.

PROOF. If such an expectation exists, $L^{\infty}(\Gamma, \mu) \otimes 1$ is Cartan and ( N,$L^{\infty}(\Gamma, \mu)$ $\otimes 1) \approx\left(\Re, L^{\infty}(\Gamma, \mu) \otimes 1\right)$ where $\Re$ is the crossed product of $L^{\infty}(\Gamma, \mu)$ by $G$. Let $\kappa$ denote the isomorphism indicated above, so $\kappa(a \otimes 1)=a \otimes 1\left(a \in L^{\infty}(\Gamma, \mu)\right)$. If $w_{g}=v_{g} \otimes \lambda(g)$ on $L^{2}(\Gamma, \mu) \otimes l^{2}(G)$, where $\lambda$ is the regular representation, we have $\kappa\left(u_{g}\right)=a_{g} w_{g}$ for some $a_{g} \in L^{\infty}(\Gamma, \mu) \otimes 1$, since $\kappa\left(u_{g}\right)$ and $w_{g}$ induce the same automorphism of $L^{\infty}(\Gamma, \mu) \otimes 1$. Clearly $a_{g h}=a_{g} \operatorname{Ad} w_{g}\left(a_{h}\right)$. By $[13,2.1 .5]$ we can write $a_{g}=a^{*} \operatorname{Ad} w_{g}(a)$ for some unitary $a \in L^{\infty}(\Gamma, \mu) \otimes l^{\infty}(G)$, and hence $\kappa\left(u_{g}\right)=$ $a^{*} w_{g} a$. So $g \rightarrow u_{g}$ is quasi-equivalent with $g \rightarrow w_{g}$, which in turn is quasi-equivalent to $\lambda$.

We remark that 4.4 gives a nontrivial example of an extension of $L^{\infty}(\mathbf{R})$ by $Z \times Z$ which is not regular in the sense of [13]. We shall also see, in 5.8, criteria similar in spirit to 4.5 which characterize those pairs $(\mathscr{R}, \alpha)$ for which $\mathscr{Q}(\Gamma) \subseteq S(\alpha)^{\prime}$ is a Cartan subalgebra.

We say that an equivalence relation $(R$ on $(\Gamma, \mu)$ is ergodic if each set $R(E)$ ( $E \subseteq \Gamma$; Borel) is either null or conull.

Proposition 4.6. Let $(\mathfrak{R}, \Theta)=S^{\prime}(\mathscr{R}, \alpha)$ with $\alpha$ replete. Then $\mathscr{N}$ is a factor if and only if $(\Re, \alpha)$ is ergodic.

Proof. Let $e \neq 0,1$ be a central projection in $\mathscr{N}$, and let $A_{1} \subseteq(\mathcal{O} e)^{\prime}, A_{2} \subseteq$ $(\mathscr{N}(1-e))^{\prime}$ be dense separable $C^{*}$-algebras. $A=A_{1} \oplus A_{2}$ is then separable and $\sigma$-weakly dense in $\mathscr{T}^{\prime}$. If $E \subseteq \Gamma$ corresponds to $e \in \mathcal{Q}$, then the decomposition of $\iota_{A}$ over $\mathbb{Q}$ has the form $\iota_{A}=\int_{E}^{\oplus} \pi_{\gamma} d \mu(\gamma) \oplus \int_{\Gamma-E}^{\oplus} \rho_{\gamma} d \nu(\gamma)$ when $\pi_{\gamma}$ has the form $\pi_{\gamma}\left(a_{1} \oplus a_{2}\right)=\tilde{\pi}_{\gamma}\left(a_{1}\right)$, and $\rho_{\gamma}$ has the form $\rho_{\gamma}\left(a_{1} \oplus a_{2}\right)=\tilde{\rho}_{\gamma}\left(a_{2}\right)$ for some representations $\tilde{\pi}_{\gamma}$ of $A_{1}$ and $\tilde{\rho}_{\gamma}$ of $A_{2}$. Since $\operatorname{ker} \pi_{\gamma} \supseteq A_{2}$, ker $\rho_{\gamma^{\prime}} \supseteq A_{1}$ for all $\gamma \in E$, $\gamma^{\prime} \in \Gamma-E$, no two of the representations $\pi_{\gamma}, \gamma \in E$, and $\rho_{\gamma^{\prime}}, \gamma^{\prime} \in \Gamma-E$, are

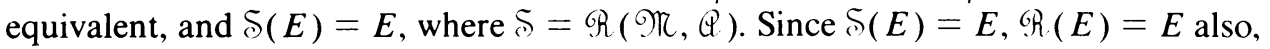
as $R \subseteq \mathcal{S}$, and $\Re$ is not ergodic.

Conversely, if $E \subseteq \Gamma$ and $\ell(E)=E$, we may clearly identify $S(\alpha)$ with $S\left(\left.\alpha\right|_{E}\right) \oplus$ $S\left(\left.\alpha\right|_{\Gamma-E}\right)$ where $\alpha_{E}$ and $\alpha_{\Gamma-E}$ are the obvious restrictions on $\left.R\right|_{E}$ and $\left.R\right|_{\Gamma-E}$. Clearly the projection $e \in Q(\Gamma)$ corresponding to $E \subseteq \Gamma$ is central in $S(\alpha)^{\prime}$.

Recall that an equivalence relation $\Re$ on $(\Gamma, \mu)$ is smooth if there is a conull Borel set $E \subseteq \Gamma$ and a Borel set $C \subseteq E$ which meets each equivalence class of $\left.R\right|_{E}$ precisely once. We refer to such a set $C$ as a section.

Proposition 4.7. Let $Q \subseteq \mathfrak{N}$ be complete. Then $\mathfrak{N}$ is type $\mathrm{I}$ if and only if $\Re(\Re, \mathbb{Q})$ is smooth.

Proof. Suppose $\Re$ is type I. Using 4.6, we may reduce to the case where $\mathbb{R}$ is a factor; thus we may assume $\mathscr{R}=\mathfrak{B}(\mathcal{H})$. Clearly then, $\mathscr{R}(\mathscr{N}, \mathscr{Q})=\Gamma \times \Gamma$ is smooth.

Conversely suppose $\mathscr{R}(\mathfrak{R}, \mathbb{Q})$ is smooth, and let $C$ be a section for $\mathscr{R}(\mathfrak{R}, \mathbb{Q})$; let $\pi: \Gamma \rightarrow C$ be the projection and $\mu=\int_{C}^{\oplus} \mu_{c} d \nu(c)$ be the decomposition of $\mu$ with 
respect to $\pi$. Standard techniques now show $S(\alpha)=\int_{C}^{\oplus} S\left(\alpha_{c}\right) d \nu(c)$ is the central decomposition of $S(\alpha)$ (using 4.6), where $\alpha_{c}$ is the restriction of $\alpha$ to $\pi^{-1}(c)$; we can thus reduce to the case where $\mathscr{R}(\mathfrak{R}, \mathscr{Q})$ is transitive on $\Gamma$. Fix $\gamma_{0} \in \Gamma$, and for $x \in \mathscr{B}\left(\mathcal{H}_{0}\right)$, define $\tilde{x} \in L^{\infty}\left(\Gamma, \mu ; \mathfrak{B}\left(\mathcal{H}_{0}\right)\right)$ by $\tilde{x}(\gamma)=\alpha\left(\gamma, \gamma_{0}\right)(x)$. Trivially, $\tilde{x} \in$ $S(\alpha)$, and the map $x \rightarrow \tilde{x}$ is a normal isomorphism of $\mathscr{B}\left(\mathcal{H}_{0}\right)$ with $S(\alpha)$, and so $\mathscr{N}$ is type I.

We note that it may well happen that $(\mathcal{O}, \mathbb{Q}) \approx S(\mathscr{R}, \alpha)$ is type I without $\mathscr{R}$ being smooth-see Example 2.5.

Recall that Zimmer in [17] has given a definition of amenability for countable, nonsingular equivalence relations $(\Re, \Gamma, \mu)$. Briefly, if $\beta$ is a representation of $R$ as isometric isomorphisms on a separable Banach space $E$, and if $\gamma \rightarrow C(\gamma)$ is a Borel field of compact convex subsets of $E^{*}$ with $a^{*}\left(\gamma, \gamma^{\prime}\right)\left(C\left(\gamma^{\prime}\right)\right)=C(\gamma)$, then there is a Borel map $\gamma \rightarrow x(\gamma) \in C(\gamma)$ with $a^{*}\left(\gamma, \gamma^{\prime}\right)\left(x\left(\gamma^{\prime}\right)\right)=x(\gamma) \mu$-a.e. Here the topology in $E^{*}$ is the $\sigma\left(E^{*}, E\right)$ topology, and $\alpha^{*}$ is the adjoint representation.

THEOREM 4.8. Let ( $R$ be countable, nonsingular and amenable, and let $\alpha$ be a replete representation of $(\stackrel{R}{R}, \Gamma, \mu)$ on $\mathrm{H}_{0}$. Then $S(\alpha)$ is an approximately finite von Neumann algebra.

Proof. We follow [17, Theorems 2.1 and 3.6] indicating only those points where changes are necessary. In particular, as in [17] it is enough to show there is a conditional expectation of $L^{\infty}\left(\Gamma, \mu ; \mathfrak{H}\left(\mathcal{H}_{0}\right)\right)$ onto $S(\alpha)$.

Let $\left\{v_{g} ; g \in G\right\}$ be as in the proof of 4.1 , so $\left(v_{g} \xi\right)(\gamma g)=a(\gamma, \gamma g) \rho(\gamma, g)^{-1 / 2} \xi(\gamma)$ for $\xi \in L^{2}\left(\Gamma, \mu ; \mathcal{H}_{0}\right)$. The operators $v_{g}$ replace the operators $\tilde{U}_{g}$ in Zimmer's proof. The only part of Zimmer's proof [17, Theorem 2.1] which requires checking is his Lemma 2.3, which we restate and prove below.

Lemma 4.9 [17, Lemma 2.3]. Let ( $R, \Gamma, \mu), \alpha, v_{g}$ be as above, and let $x \in$ $L^{\infty}\left(\Gamma, \mu ; \mathfrak{B}\left(\mathcal{H}_{0}\right)\right)$. Let $C_{\Gamma}(x)$ be the weakly closed convex hull of $\left\{\sum_{1}^{n} f_{i} v_{g_{i}} \times v_{g_{i}}^{*}\right.$ : $f_{i} \in \mathbb{Q}(\Gamma), f_{i} \geqslant 0, \Sigma_{1}^{n} f_{i}=1$, and $\left.g_{i} \in G, i=1, \ldots, n\right\}$. Then $C_{\Gamma}(x) \cap\left\{v_{g}\right\}^{\prime} \neq \varnothing$.

Proof. Let $x \sim x(\gamma)$, and define $x_{g}(\gamma)=\alpha\left(\gamma, \gamma g^{-1}\right)\left(x\left(\gamma g^{-1}\right)\right)$; in fact $x_{g}=$ $v_{g} x v_{g}^{*}$. By Lemma 2.2 of [17], $C_{\gamma}(x)=$ weakly closed convex hull of $\left\{x_{g}(\gamma): g \in G\right\}$ is weakly compact, and $\gamma \rightarrow C_{\gamma}(x)$ is a Borel field. Furthermore, for $h \in G$, we have

$$
\begin{aligned}
\alpha(\gamma h, \gamma)\left(x_{g}(\gamma)\right) & =\alpha(\gamma h, \gamma) \alpha\left(\gamma, \gamma g^{-1}\right)(x(\gamma)) \\
& =\alpha\left(\gamma h, \gamma g^{-1}\right)(x(\gamma))=x_{g}(\gamma h)
\end{aligned}
$$

so that $\alpha\left(\gamma, \gamma^{\prime}\right)\left(C\left(\gamma^{\prime}\right)\right)=C(\gamma)$ on some i.r. of $\mathcal{R}$. Finally, $\alpha$ is a dual representation, for if we let $\mathcal{T} \mathcal{C}$ denote the trace class operators on $\mathcal{H}_{0}$, and identify $\mathscr{B}\left(\mathcal{H}_{0}\right)$ with Tৎ* we may define $\alpha_{*}$ by $\left\langle\alpha_{*}\left(\gamma, \gamma^{\prime}\right)(T), x\right\rangle=\left\langle T, \alpha\left(\gamma, \gamma^{\prime}\right)^{-1}(x)\right\rangle$ for $T \in \mathcal{T} \bigodot$ and $x \in \mathfrak{B}\left(\mathcal{H}_{0}\right)$, so that $\left(\boldsymbol{\alpha}_{*}\right)^{*}=\alpha$. Thus there is, by amenability, a Borel map $\gamma \rightarrow y(\gamma)$ $\in C_{\gamma}(x)$ with $\alpha\left(\gamma, \gamma^{\prime}\right)\left(y\left(\gamma^{\prime}\right)\right)=y(\gamma) \mu$-a.e. Since $\|y(\gamma)\|$ is essentially bounded, $y \in S(\alpha)$ and $y \in\left\{u_{g}\right\}^{\prime}$. The proof that $y \in C_{\Gamma}(x)$ is exactly as in [17].

The remainder of the proof of 4.8 follows exactly as in [17]. 
5. Cohomology and dominance. Let $(\Re, \Gamma, \mu)$ be an analytic equivalence relation; a Borel map $\omega: \mathscr{R}^{(2)}=\left\{\left(\gamma_{1}, \gamma_{2}, \gamma_{3}\right):\left(\gamma_{1}, \gamma_{2}\right) \in \mathcal{R},\left(\gamma_{2}, \gamma_{3}\right) \in \Re\right\} \rightarrow \mathbf{T}$ will be termed a 2-cocycle if $\omega\left(\gamma_{2}, \gamma_{3}, \gamma_{4}\right) \omega\left(\gamma_{1}, \gamma_{2}, \gamma_{4}\right)=\omega\left(\gamma_{1}, \gamma_{3}, \gamma_{4}\right) \omega\left(\gamma_{1}, \gamma_{2}, \gamma_{3}\right)$ on $\left.\Re^{(2)}\right|_{E}=$ $\left\{\left(\gamma_{1}, \gamma_{2}, \gamma_{3}\right) \in \mathfrak{R}^{(2)}: \gamma_{1}, \gamma_{2}, \gamma_{3} \in E\right\}$ where $E$ is conull and Borel. A cocycle $\omega$ is reduced if $\omega\left(\gamma_{1}, \gamma_{2}, \gamma_{3}\right)=1$ whenever $\gamma_{1}=\gamma_{2}$ or $\gamma_{2}=\gamma_{3}$, and we identify cocycles if they agree on some $\left.\mathcal{R}^{(2)}\right|_{E}$ where $\mu(\Gamma-E)=0$. The group of all reduced cocycles is denoted $Z^{2}(\Re, \mathbf{T})$. A cocycle $\omega$ is a coboundary if $\omega\left(\gamma_{1}, \gamma_{2}, \gamma_{3}\right)=$ $b\left(\gamma_{1}, \gamma_{2}\right) b\left(\gamma_{1}, \gamma_{3}\right)^{-1} b\left(\gamma_{2}, \gamma_{3}\right)$ for some Borel map $b: \Omega \rightarrow \mathbf{T}$, and in this case we write $\omega=\partial b$. The group of all coboundaries is denoted $B^{2}(\Re, \mathbf{T})$, and the quotient $Z^{2}(\Re, \mathbf{T}) / B^{2}(\Re, \mathbf{T})$ by $H^{2}(\Re, \mathbf{T})$. The reader will find an extensive development of the cohomology of countable nonsingular equivalence relations in [4].

If $\Phi \in \operatorname{Aut}(\Re, \mu)$ and $\omega \in Z^{2}(\mathscr{R}, \mathbf{T})$ then with $\Phi(\omega)\left(\gamma_{1}, \gamma_{2}, \gamma_{3}\right)=$

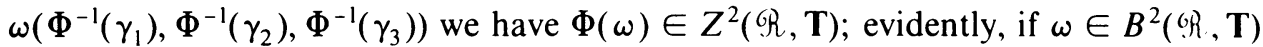
then $\Phi(\omega) \in B^{2}(R, \mathbf{T})$ and we have a well-defined action of $\operatorname{Aut}(R, \mu)$ on $H^{2}(R, \mathbf{T})$.

If $\alpha$ is a representation of $(\Re, \Gamma, \mu)$, choose a Borel map $a: \mathscr{R} \rightarrow \mathfrak{H}\left(\mathcal{H}_{0}\right)$ with unitary values and $\alpha\left(\gamma_{1}, \gamma_{2}\right)=\operatorname{Ad} a\left(\gamma_{1}, \gamma_{2}\right)$ on some i.r. $\left.{ }^{\circ}\right|_{l}$; we write $\alpha=\operatorname{Ad} a$. Since $\alpha\left(\gamma_{1}, \gamma_{2}\right) \alpha\left(\gamma_{2}, \gamma_{3}\right)=\alpha\left(\gamma_{1}, \gamma_{3}\right)$ we have $a\left(\gamma_{1}, \gamma_{2}\right) a\left(\gamma_{2}, \gamma_{3}\right) \omega\left(\gamma_{1}, \gamma_{2}, \gamma_{3}\right)=$ $a\left(\gamma_{1}, \gamma_{3}\right)$ for some Borel map $\omega:\left.G^{(2)}\right|_{E} \rightarrow \mathbf{T}$.

Proposition 5.1. With notation as above

(i) $\omega \in Z^{2}(\Re, \mathbf{T})$, and the class of $\omega$ in $H^{2}(\Re, \mathbf{T})$ depends only on the conjugacy class of $\alpha$; we write this class as $\partial \alpha$ or $\partial$ a, as convenient.

(ii) If $\alpha=\beta \cdot \Phi^{-1}$ for some $\Phi \in \operatorname{Aut}($ GR, $\mu)$, then $\partial \alpha=\Phi(\partial \beta)$.

(iii) If $\alpha, \beta$ are representations of $($ ( $R, \Gamma, \mu)$ then $\partial(\alpha \otimes \beta)=\partial \alpha \cdot \partial \beta$.

Proof. (i) That $\omega \in Z^{2}\left(\sigma_{R}, \mathbf{T}\right)$ results from the equality

$$
\left(a\left(\gamma_{1}, \gamma_{2}\right) a\left(\gamma_{2}, \gamma_{3}\right)\right) a\left(\gamma_{3}, \gamma_{4}\right)=a\left(\gamma_{1}, \gamma_{2}\right)\left(a\left(\gamma_{2}, \gamma_{3}\right) a\left(\gamma_{3}, \gamma_{4}\right)\right)
$$

on $\left.R\right|_{E}$

If $\alpha=\operatorname{Ad} b$ for some unitary Borel map $b:$ oR $\left.\right|_{E} \rightarrow \mathfrak{B}\left(\mathcal{H}_{0}\right)$, then $a\left(\gamma_{1}, \gamma_{2}\right)=$

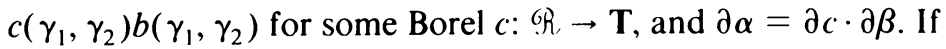

$$
\alpha\left(\gamma, \gamma^{\prime}\right) \otimes \mathrm{id}=\operatorname{Ad} u(\gamma)\left(\beta\left(\gamma, \gamma^{\prime}\right) \otimes \mathrm{id}\right) \operatorname{Ad} u\left(\gamma^{\prime}\right)^{*},
$$

then

$$
a\left(\gamma_{1}, \gamma_{2}\right) \otimes 1=c\left(\gamma_{1}, \gamma_{2}\right) u\left(\gamma_{1}\right)\left(b\left(\gamma_{1}, \gamma_{2}\right) \otimes 1\right) u\left(\gamma_{2}\right)^{*}
$$

where $\alpha=\operatorname{Ad} a, \beta=\operatorname{Ad} b$, and $c:\left.\stackrel{\leftrightarrow}{R}\right|_{t:} \rightarrow \mathbf{T}$ is Borel; again $\partial \alpha=\partial c \cdot \partial \beta$.

(ii) and (iii) are trivial and left to the reader.

Definition 5.2. (i) The orbit under $\operatorname{Aut}(G R, \mu)$ in $H^{2}(G R, \mathbf{T})$ determined by the quasi-equivalence of a representation $(\Re, \alpha)$ will be denoted $\Delta(\Re, \alpha)$, and termed the fundamental class of $(\Re, \alpha)$.

(ii) If $\mathscr{Q} \subseteq \Re$ is maximal abelian, we set $\Delta(\mathscr{R}, \mathbb{Q})=\Delta(\mathscr{T}(\mathfrak{T}, \mathbb{Q}))$.

Definition 5.3. A representation $(\Re, \alpha)$ is flat if $\Delta(\Re, \alpha)$ is trivial in $H^{2}(\Re, \mathbf{T})$. A pair $(\mathfrak{N}, \mathbb{Q})$ is flat if $\mathscr{T}(\mathfrak{N}, \mathbb{Q})$ is flat.

It seems difficult to establish the existence of representations with a specified fundamental class, except in the special case of "measured equivalence relations" in 
the terminology of [6], or of "standard Borel principal groupoids with transverse measure and proper transverse function" in the terminology of [2]. Despite the manifest advantages of Connes viewpoint [2], we will adopt that of [6] since it is closer to the needs of the remainder of the paper, and since many of the results to which we will need to appeal appear in the literature only in this language.

According to [6], a principal measured groupoid is a system $(R, \Gamma, \mu, \nu)$, where $(\mathscr{R}, \Gamma, \mu)$ is an analytic equivalence relation, and $\nu$ is a measure on $R$ with properties given below. Let $r: G \rightarrow \Gamma, r\left(\gamma, \gamma^{\prime}\right)=\gamma$, and let $\nu=\int_{\Gamma}^{\oplus} \nu_{\gamma} d \tilde{\nu}(\gamma)$ be the decomposition of $\nu$ with respect to $r$. Note that if $\left(\gamma, \gamma^{\prime}\right) \in \mathscr{R}$, there is an associated transformation $\tau\left(\gamma, \gamma^{\prime}\right): r^{-1}\left(\gamma^{\prime}\right) \rightarrow r^{-1}(\gamma)$ given by $\left(\tau\left(\gamma, \gamma^{\prime}\right)\right)\left(\gamma^{\prime}, \gamma^{\prime \prime}\right)=\left(\gamma, \gamma^{\prime \prime}\right)$. For $(\Re, \Gamma, \mu, \nu)$ to be a measured groupoid we demand that

(i) $\nu_{\gamma} \cdot \tau\left(\gamma, \gamma^{\prime}\right)$ is equivalent to $\nu_{\gamma^{\prime}}$ on some i.r.

(ii) $\nu\left(E^{-1}\right)=\nu(E)$ for $E \subseteq \Gamma$ Borel, where $E^{-1}=\left\{\left(\gamma, \gamma^{\prime}\right):\left(\gamma^{\prime}, \gamma\right) \in E\right\}$.

(iii) $\tilde{\nu}$ is equivalent to $\mu$.

The reader should consult $[6,7,4]$ for a more complete discussion. In particular, it is shown in [6] that we may change $\nu$ within its equivalence class to attain $\nu_{\gamma} \cdot \tau\left(\gamma, \gamma^{\prime}\right)=\nu_{\gamma^{\prime}}$ on some i.r., while maintaining (iii) but not (ii). We refer to $(\nu, \tilde{\nu})$ as a Haar measure for $\mathcal{R}$. All equivalence relations arising from nonsingular actions of second countable locally compact groups admit natural structures as measured groupoids; if $R$ is countable nonsingular, the measures $\nu_{\gamma}$ above may be taken as counting measures.

The "dictionary" between the language above and that of [2] is the following; the family $\left\{\nu_{\gamma}: \gamma \in \Gamma\right\}$ of measures above (satisfying the invariance condition) is precisely what Connes calls a transverse function; the measure $\tilde{v}$ above is nothing but Connes' measure $\Lambda_{\nu}$ on $\Gamma$ determined by some transverse measure $\Lambda$ on $R$. One of the principal differences between the theories lies in the treatment of null sets, those of Connes being saturated and $\Lambda_{\nu}$-null, while those of Hahn are simply $\tilde{\nu}$ - (or $\mu-)$ null.

In what follows we construct what effectively are the concrete projective analogue of Connes' "square integrable" representations in a different language. Essentially all of Connes' results for square integrable representations persist for projective representations; the only one which we need subsequently is proven below (Theorem 5.7), and we leave the remainder to the interested reader.

EXAMPLE 5.4. Let $\Re$ be a principal measured groupoid with Haar measure $(\nu, \mu)$, and decomposition $\nu=\int_{\rho}^{\oplus} \nu_{\gamma} d \mu(\gamma)$. Note that for $\xi \in L^{2}(\Re, \nu)$, if $\xi \sim \xi(\gamma)$ in the decomposition $L^{2}(\Re, \nu)=\int_{\rho}^{\oplus} L^{2}\left(r^{-1}(\gamma), \nu_{\gamma}\right) d \mu(\gamma)$, then we may take $(\xi(\gamma))\left(\gamma^{\prime}\right)=$ $\xi\left(\gamma, \gamma^{\prime}\right)$ for $\gamma^{\prime} \in r^{-1}(\gamma)$.

Set $\mathcal{H}(\gamma)=L^{2}\left(r^{-1}(\gamma), \nu_{\gamma}\right)$; considering if necessary $L^{2}(\mathscr{R}, \nu) \otimes \mathcal{K}_{0}$, we may assume $\mathcal{H}(\gamma)$ is isomorphic with $\mathcal{H}_{0}$ for all $\gamma \in \Gamma$.

Let $\omega \in Z^{2}(\mathscr{R}, \mathbf{T})$ and define unitaries $t^{\omega}\left(\gamma_{1}, \gamma_{2}\right): \mathcal{H}\left(\gamma_{2}\right) \rightarrow \mathcal{H}\left(\gamma_{1}\right)$ by $\left(t^{\omega}\left(\gamma_{1}, \gamma_{2}\right) \xi\right)(\gamma)=\omega\left(\gamma, \gamma_{1}, \gamma_{2}\right)^{-1} \xi(\gamma)$, for $\left(\gamma_{1}, \gamma_{2}\right) \in \Re$ and $\gamma \in r^{-1}\left(\gamma_{1}\right)$. (Note: this is the unitary $W_{\sigma}$ of [7, Example 3.11], with the identification $\sigma\left(\left(\gamma_{1}, \gamma_{2}\right),\left(\gamma_{2}, \gamma_{3}\right)\right)=$ $\omega\left(\gamma_{1}, \gamma_{2}, \gamma_{3}\right)$.) The fact that $t^{\omega}\left(\gamma_{1}, \gamma_{2}\right)$ is unitary is equivalent to the property $\nu_{\gamma} \cdot \tau\left(\gamma, \gamma^{\prime}\right)=\nu_{\gamma^{\prime}}$ of Haar measure. Also, for $\xi \in \mathcal{H}\left(\gamma_{3}\right)$, we have 


$$
\begin{aligned}
\left(t^{\omega}\left(\gamma_{1}, \gamma_{2}\right) t^{\omega}\left(\gamma_{2}, \gamma_{3}\right) \xi\right)(\gamma) & =\omega\left(\gamma, \gamma_{1}, \gamma_{2}\right)^{-1} \omega\left(\gamma, \gamma_{2}, \gamma_{3}\right)^{-1} \xi(\gamma) \\
& =\omega\left(\gamma_{1}, \gamma_{2}, \gamma_{3}\right)^{-1} \omega\left(\gamma, \gamma_{1}, \gamma_{3}\right)^{-1} \xi(\gamma) \\
& =\omega\left(\gamma_{1}, \gamma_{2}, \gamma_{3}\right)^{-1}\left(t^{\omega}\left(\gamma_{1}, \gamma_{3}\right) \xi\right)(\gamma)
\end{aligned}
$$

so that $\omega\left(\gamma_{1}, \gamma_{2}, \gamma_{3}\right) t^{\omega}\left(\gamma_{1}, \gamma_{2}\right) t^{\omega}\left(\gamma_{2}, \gamma_{3}\right)=t^{\omega}\left(\gamma_{1}, \gamma_{3}\right)$ and the map $\left(\gamma, \gamma^{\prime}\right) \in \mathscr{R} \rightarrow$ $\tau^{\omega}\left(\gamma, \gamma^{\prime}\right)=\operatorname{Ad} t^{\omega}\left(\gamma, \gamma^{\prime}\right)$ is a representation of $R$ in $\mathcal{H}_{0}^{\prime}$.

Proposition 5.5. With notation as above

(i) $S^{\prime}\left(R, \tau^{\omega}\right)=\left\{\lambda^{\omega}(R)^{\prime \prime}, \mathbb{Q}(\Gamma)\right\}$, where $\lambda^{\omega}$ is the left regular $\omega$-representation of ( $R, \Gamma, \mu)$.

(ii) $\Delta\left(\Re, \tau^{\omega}\right)=\Delta\left(S^{\prime}\left(\Re, \tau^{\omega}\right)\right)$ is the class determined by $\omega$.

Proof. (i) is the content of [7, Theorem 4.3]. Note also that $\tau^{\omega}$ is replete, by [7, Theorem 5.1].

(ii) That $\Delta\left(G R, \tau^{\omega}\right)$ is the class determined by $\omega$ follows from the above computation; to establish that $\Delta\left(S^{\prime}\left(\Re, \tau^{\omega}\right)\right)=\Delta\left(\Re, \tau^{\omega}\right)$ it is enough to show $\widetilde{T}\left(S^{\prime}\left(\Re, \tau^{\omega}\right)\right)$ $=\left(\Re, \tau^{\omega}\right)$ is complete.

Proposition 3.2 shows that $\mathcal{T}\left(S^{\prime}\left(\Re, \tau^{\omega}\right)\right)$ extends $\left(\Re, \tau^{\omega}\right)$, although this may be checked directly using the fact that $S\left(\Re, \tau^{\omega}\right)$ is generated by operators

$$
\left(\pi^{\omega}(f) \xi\right)\left(\gamma_{1}, \gamma_{2}\right) \int f\left(\gamma_{2}, \gamma\right) \delta\left(\gamma_{2}, \gamma\right)^{1 / 2} \omega\left(\gamma_{2}, \gamma, \gamma_{3}\right) \xi\left(\gamma_{1}, \gamma\right) d \nu_{\gamma_{2}}(\gamma)
$$

on $L^{2}(\Re, \nu)$, where $\delta$ is the modular function of $(\mathcal{R}, \Gamma, \mu, \nu)$ and $f$ is a suitably restricted complex valued Borel function on $\mathcal{R}$ (see [7, Theorem 4.3] and the discussion preceding it). The decomposition of $\pi^{\omega}$ over $\mathscr{Q}(\Gamma)$ is given then by representations $\pi_{\gamma}^{\omega}$ on $\mathcal{H}(\gamma)$ of the form

$$
\left(\pi_{\gamma_{1}}^{\omega}(f) \xi\right)\left(\gamma_{2}\right)=\int f\left(\gamma_{2}, \gamma\right) \delta\left(\gamma_{2}, \gamma\right)^{1 / 2} \omega\left(\gamma_{2}, \gamma, \gamma_{1}\right) \xi(\gamma) d \nu_{\gamma_{2}}(\gamma)
$$

Note that $S\left(\mathcal{R}, \tau^{\omega}\right)$ contains a copy of $L^{\infty}(\Gamma, \mu)$, this time acting via operators $D_{\varphi}, \varphi \in L^{\infty}(\Gamma, \mu)$, where $\left(D_{\varphi} \xi\right)\left(\gamma, \gamma^{\prime}\right)=\varphi\left(\gamma^{\prime}\right) \xi\left(\gamma, \gamma^{\prime}\right)$ on $L^{2}(\stackrel{\leftrightarrow}{R}, \nu)$. If $D_{\varphi} \sim D_{\varphi}(\gamma)$ in the decomposition over $\mathbb{Q}(\Gamma),\left(D_{\varphi}(\gamma) \xi\right)\left(\gamma^{\prime}\right)=\varphi\left(\gamma^{\prime}\right) \xi\left(\gamma^{\prime}\right)$. Let $A$ be a weakly dense separable $C^{*}$-subalgebra of $S\left(\Re, \tau^{\omega}\right)$ containing the characteristic functions of a countable separating family of Borel sets in $\Gamma$. If $u$ is a unitary which intertwines $\pi_{\gamma}^{A}$ and $\pi_{\gamma^{\prime}}^{A}$, then $u$ is of the form $(u \xi)\left(\gamma_{1}\right)=c\left(\gamma_{1}\right) \xi\left(\Phi\left(\gamma_{1}\right)\right)$ for $\xi \in \mathcal{H}(\gamma)$, where $\Phi$ : $\left(r^{-1}(\gamma), \nu_{\gamma}\right) \rightarrow\left(\mathrm{r}^{-1}\left(\gamma^{\prime}\right), \nu_{\gamma^{\prime}}\right)$ is an isomorphism and $c: r^{-1}(\gamma) \rightarrow \mathbf{C}$ is Borel, since Ad $u$ carries $L^{\infty}\left(r^{-1}(\gamma), \nu_{\gamma}\right)$ to $L^{\infty}\left(r^{-1}\left(\gamma^{\prime}\right), \nu_{\gamma^{\prime}}\right)$. But

$$
\left(u \pi_{\gamma^{\prime}}^{A}\left(D_{\varphi}\right) \xi\right)\left(\gamma_{1}\right)=c\left(\gamma_{1}\right) \varphi\left(\Phi\left(\gamma_{1}\right)\right) \xi\left(\Phi\left(\gamma_{1}\right)\right)
$$

and

$$
\left(\pi_{\gamma}^{A}\left(D_{\varphi}\right) u \xi\right)\left(\gamma_{1}\right)=\varphi\left(\gamma_{1}\right) c\left(\gamma_{1}\right) \xi\left(\Phi\left(\gamma_{1}\right)\right)
$$

so $\varphi\left(\gamma_{1}\right)=\varphi\left(\Phi\left(\gamma_{1}\right)\right)$ for all $\varphi$ in some separating family, and $\Phi$ is the identity; thus $\left(\gamma, \gamma^{\prime}\right) \in \mathcal{R}$ and $\left(\mathcal{R}, \tau^{\omega}\right)$ is complete as required.

The representations $\tau^{\omega}$ constructed above have many interesting properties. 
Definition 5.6. Let $(\mathcal{R}, \alpha)$ be a representation. $\alpha$ is said to be dominant if for all flat representations $(\Re, \beta), \alpha^{*} \otimes \beta$ is quasi-equivalent to $\alpha$.

The following theorem is not surprising in view of the known facts concerning the regular representation of locally compact groups-see also [3, §6, Theorem 10].

THEOREM 5.7. Let ( $R, \Gamma, \mu, \nu)$ be a principal measured groupoid, and $\alpha$ a representation of $\Re$ on $\mathcal{H}_{0}$, with $\omega \in \Delta(\Re, \alpha)$. Then $\alpha$ is dominant if and only if $\alpha$ is quasi-equivalent to $\tau^{\omega}$.

Proof. It is sufficient to show that for $\sigma \in Z^{2}(\mathscr{G}, \mathbf{T})$, and for $\alpha$ a representation of $\Re$ with $\partial \alpha=\omega$, we have $\tau^{\sigma} \otimes \alpha \approx \tau^{\sigma \omega}$. For this then implies that if $\alpha$ is dominant, $\alpha \approx \tau \otimes \alpha \approx \tau^{\omega}$, and, assuming $\alpha$ is flat, $\tau^{\sigma} \otimes \alpha \approx \tau^{\sigma}$ is dominant.

Let $\alpha=\operatorname{Ad} a$, with $\partial \alpha=\omega$, and let $\tau^{\sigma}=\operatorname{Ad} t^{\sigma}$ be as in Example 5.4, for $\sigma \in Z^{2}(\mathcal{R}, \mathbf{T})$. So $\tau^{\sigma} \otimes \alpha=\operatorname{Ad}\left(t^{\sigma} \otimes a\right)$. Define a unitary $u(\gamma)$ on $L^{2}\left(r^{-1}(\gamma), \nu_{\gamma} ; \mathcal{H}_{0}\right)$ by $(u(\gamma) \xi)\left(\gamma^{\prime}\right)=a\left(\gamma^{\prime}, \gamma\right) \xi\left(\gamma^{\prime}\right)$. Then we compute

$$
\begin{aligned}
\left(u\left(\gamma_{1}\right)\left(t^{\sigma}\left(\gamma_{1}, \gamma_{2}\right) \otimes a\left(\gamma_{1}, \gamma_{2}\right)\right) u\left(\gamma_{2}\right)^{*} \xi\right)(\gamma) & \\
& =a\left(\gamma, \gamma_{1}\right) \omega\left(\gamma, \gamma_{1}, \gamma_{2}\right)^{-1} a\left(\gamma_{1}, \gamma_{2}\right) a\left(\gamma, \gamma_{2}\right)^{*} \xi(\gamma) \\
& =(\partial \alpha)\left(\gamma, \gamma_{1}, \gamma_{2}\right)^{-1} \omega\left(\gamma, \gamma_{1}, \gamma_{2}\right)^{-1} \xi(\gamma) \\
& =\left(\left(t^{\omega \sigma}\left(\gamma_{1}, \gamma_{2}\right) \otimes 1\right) \xi\right)(\gamma) .
\end{aligned}
$$

Thus $\tau^{\sigma} \otimes \alpha \approx \tau^{\sigma \omega}$ as required.

COROLlaRY 5.8. Let $\mathbb{Q} \subseteq \mathfrak{M}$ be complete and maximal abelian. Then $\mathbb{Q}$ is Cartan if and only if $\mathscr{R}(\mathfrak{T}, \mathbb{Q})$ is countable nonsingular, and $\mathcal{T}(\mathfrak{T}, \mathbb{Q}) \approx\left(\Re(\mathscr{N}, \mathbb{Q}), \tau^{\omega}\right)$ where $\omega \in \Delta(\mathscr{T}, \mathbb{Q})$.

Proof. It $Q$ is Cartan, then $\mathscr{R}(\mathscr{T}, \mathcal{Q})$ is countable nonsingular by [8]. From [5] $(\Re, Q)$ is generated from the projective regular representation of $\Re=\Re(\Re, \mathbb{Q})$ for some cocycle $\sigma$, so by $5.5(1),(\Re, Q) \approx S^{\prime}\left(\Re, \tau^{\sigma}\right)$ for some $\sigma \in Z^{2}(\Re, T)$. But it is straightforward to check that $\sigma \in \Delta(\mathfrak{R}, \mathbb{Q})$.

The converse follows by [7, Theorem 4.3], as $\mathbb{Q}$ is complete.

Corollary 5.9. Let $Q \subseteq \Re, \mathscr{M} \subseteq \Re$ be Cartan subalgebras. Then the following are equivalent:

(i) $(\mathscr{N}, Q) \approx(\mathscr{K}, \Re)$,

(ii) $\mathscr{T}(\mathscr{T}, \mathbb{Q}) \approx \sigma(\mathscr{T}, \mathscr{B})$,

(iii) $\Delta(\mathfrak{R}, \mathbb{Q})=\Delta(\mathfrak{N}, \mathscr{B})$.

6. Galois theory. Let $(\Re, \alpha)$ be a complete, replete representation, and set $(\mathscr{R}, \mathbb{Q})=S^{\prime}(\Re, \alpha)$. We wish to demonstrate a lattice isomorphism between equivalence relations $\mathcal{S} \subseteq \Re$ with $(\mathcal{S}, \alpha)$ complete, and von Neumann subalgebras $\Re \subseteq \Re$ containing $\mathcal{Q}$, and for which $(\mathcal{T}, \mathbb{Q})$ is complete.

Let $\delta_{1}, \mathcal{S}_{2} \subseteq \Re$ be equivalence relations, with $\mathcal{S}_{1} \wedge \mathcal{S}_{2}=\mathcal{S}_{1} \cap \mathcal{S}_{2}$, and let $\left(\mathcal{S}_{1} \vee\right.$ $\left.\delta_{2}, \alpha\right)$ be the completion of $(\delta, \alpha)$, where $\delta$ is the equivalence relation generated by $\delta_{1}$ and $\delta_{2}$. (Simple examples show that $\delta$ may fail to be complete even if $\delta_{1}$ and $\delta_{2}$ are complete.) Similarly for von Neumann algebras $\Re_{1}, \Re_{2}$ with $\mathbb{Q} \subseteq \Re_{j} \subseteq \Re$, 
$j=1,2$, let $\mathfrak{K}_{1} \vee \Re_{2}$ denote the algebra which they generate, and $\left(\Re_{1} \wedge \Re_{2}, \mathscr{Q}\right)$ the completion of $\left(\Re_{1} \cap \Re_{2}, \mathbb{Q}\right)$.

LEMMA 6.1. (i) If $\left(\S_{j}, \alpha\right), j=1,2$, are complete, so is $\left(\delta_{1} \wedge \delta_{2}, \alpha\right)$.

(ii) If $\left(\Re_{j}, \mathbb{Q}\right), j=1,2$, are complete, so is $\left(\mathfrak{X}_{1} \vee \mathfrak{K}_{2}, \mathbb{Q}\right)$.

Proof. (i) Let $A_{j}, j=1,2$, be dense separable $C^{*}$-subalgebras of $S^{\prime}\left(\delta_{j}, \alpha\right)$ respectively, and let $A \subseteq S^{\prime}\left(\mathcal{S}_{1} \cap \mathcal{S}_{2}, \alpha\right)$ be dense and separable. Clearly we may assume that $A_{j} \subseteq A, j=1,2$, and hence that

$$
\Re\left(S\left(\delta_{1} \cap \delta_{2}\right)^{\prime}, \mathbb{Q}(\Gamma)\right) \subseteq \Re\left(S\left(\delta_{1}\right)^{\prime}, \mathbb{Q}(\Gamma)\right) \cap \mathcal{R}\left(S\left(\delta_{2}\right)^{\prime}, \mathbb{Q}(\Gamma)\right) .
$$

Thus if $\mathcal{S}_{1}$ and $\mathcal{S}_{2}$ are complete $\mathscr{R}\left(S\left(\mathcal{S}_{1} \cap \mathcal{S}_{2}\right)^{\prime}, \mathbb{Q}(\Gamma)\right) \subseteq \mathcal{S}_{1} \cap \mathcal{S}_{2}$. The reverse inclusion is automatic by 3.2 , and so $\delta_{1} \wedge \delta_{2}$ is complete if $\delta_{1}$ and $\delta_{2}$ are.

(ii) Let $\left(\Re_{j}, \mathscr{Q}\right)$ be complete, and $\delta_{j}=\mathscr{R}\left(\Re_{j}, \mathscr{Q}\right), j=1,2$, so that $\Re_{j}=S\left(\delta_{j}, \alpha\right)^{\prime}$, $j=1,2$. The desired result now follows from the obvious relation $S\left(\delta_{1} \vee \S_{2}, \alpha\right)=$ $S\left(\delta_{1}, \alpha\right) \cap S\left(\delta_{2}, \alpha\right)$, which holds for arbitrary subequivalence relations $\S_{1}$ and $\delta_{2}$.

Proposition 6.2. Let $(\Re, \alpha)$ be complete and replete. Then the map $\mathcal{\subseteq} \Re \rightarrow$ $S(\mathcal{S}, \alpha)^{\prime}$ determines a lattice isomorphism between the set of subequivalence relations $\delta$ of $\Re$ with $(\delta, \alpha)$ complete (identified up to null sets), and the set of von Neumann subalgebras $\Re \subseteq \Re$ with $(\Re, Q)$ complete. Thus for complete $\mathcal{S}_{1}, \mathcal{S}_{2} \subseteq \Re$, we have

$$
S\left(\varsigma_{1} \wedge \varsigma_{2}, \alpha\right)^{\prime}=S\left(\S_{1}, \alpha\right)^{\prime} \wedge S\left(\S_{2}, \alpha\right)^{\prime}
$$

and

$$
S\left(\delta_{1} \vee \delta_{2}, \alpha\right)^{\prime}=S\left(\delta_{1}, \alpha\right)^{\prime} \vee S\left(\delta_{2}, \alpha\right)^{\prime}
$$

Proof. Since we restrict to complete subrelations and subalgebras, the indicated map is a bijection. That it is a lattice isomorphism follows from 6.1.

The above Galois correspondence is well known for crossed products of abelian algebras by discrete groups, and for some other cases as well (see $[1,16])$.

\section{REFERENCES}

1. A. Connes, Une classification des facteurs de type III, Ann. École Norm. Sup. (4) 6(1973), 18-252.

2. Sur la théorie non-commutative de l'intégration, Lecture Notes in Math., vol. 725. Springer-Verlag, Berlin and New York.

3. H. A. Dye, On groups of measure preserving transformations. I, Amer. J. Math. 81 (1959), 119-159.

4. J. Feldman and C. C. Moore, Ergodic equivalence relations, von Neumann algebras and cohomologv, I, Trans. Amer. Math. Soc. 234 (1977), 289-324.

5. Ergodic equivalence relations, von Neumann algebras and cohomologv, II, Trans. Amer. Math. Soc. 234 (1977), 325-359.

6. P. Hahn, Haur measure for measure groupoids, Trans. Amer. Math. Soc. 242 (1978), 1-34.

7. $\quad$ The regular representation of measure groupoids, Trans. Amer. Math. Soc. 242 (1978), 35-73.

8. Reconstruction of a factor from measures on Takesaki's unitary equivalence relation, J. Funct. Anal. 31 (1979), 263-271.

9. A. A. Kirillov, Elements of the theory of representations, Die Grundlehren der Math. Wissenschaften, No. 220, Springer-Verlag, Berlin and New York, 1976.

10. G. Mackey, Ergodicity in the theory of group representations, Proceedings of the I.C.M. 2 (1970), 401-405.

11. B_ Borel structures in groups and their duals, Trans. Amer. Math. Soc. 80 (1957), 134-165.

12. __ Point realization of transformation groups, Illinois J. Math. 6 (1962), 327-335. 
13. C. E. Sutherland, Cohomology and extensions of von Neumann algebras. II, R.I.M.S., Kyoto Univ. 16 (1980), 135-174.

14. M. Takesaki, On the unitary equivalence among components of decompositions of representations of involutive Banach algebras, and the associated diagonal algebras, Tôhoku Math. J. 15 (1963), 365-393.

15. Duality for crossed products and the structure of von Neumann algebras of type III. Acta Math. 131 (1974), 249-310.

16. M. Takesaki and Y. Nakagami, Duality for crossed products of von Neumann algebras, Lecture Notes in Math., vol. 731, Springer-Verlag, Berlin and New York.

17. R. J. Zimmer, Hyperfinite factors and amenable ergodic actions, Invent. Math. 41 (1977), 23-31.

Department of Mathematics, University of New South Wales, Kensington 2033, Australia 